Please do not remove this page

RMIT

UNIVERSITY

\title{
Tunable Biomimetic Hydrogels from Silk Fibroin and Nanocellulose
}

Dorishetty, Pramod; Balu, Rajkamal; Athukoralalage, Sandya; Greaves, Tamar; Mata, Jitendra; de Campo, Liliana; Saha, Nabanita

https://researchrepository.rmit.edu.au/esploro/outputs/9921861395201341/filesAndLinks?institution=61RMIT_INST\&index=null

Dorishetty, P., Balu, R., Athukoralalage, S., Greaves, T., Mata, J., de Campo, L., Saha, N., Zannettino, A., Dutta, N., \& Choudhury, N. (2020). Tunable Biomimetic Hydrogels from Silk Fibroin and Nanocellulose. ACS Sustainable Chemistry and Engineering, 8(6), 2375-2389.

https://doi.org/10.1021/acssuschemeng.9b05317

Document Version: Accepted Manuscript

Published Version: https://doi.org/10.1021/acssuschemeng.9b05317

Repository homepage: https://researchrepository.rmit.edu.au

(c) 2020 American Chemical Society

Downloaded On 2023/04/26 22:18:56 +1000 


\section{Tunable Biomimetic Hydrogels from Silk Fibroin and Nanocellulose}

Pramod Dorishetty, ${ }^{a}$ Rajkamal Balu, ${ }^{a}$ Sandya S. Athukoralalage, ${ }^{a}$ Tamar L. Greaves, ${ }^{\mathrm{b}}$ Jitendra P. Mata, ${ }^{\mathrm{c}}$ Liliana de Campo, ${ }^{\mathrm{c}}$ Nabanita Saha, ${ }^{\mathrm{d}}$ Andrew C. W. Zannettino, ${ }^{\mathrm{e}}$ Naba K. Dutta*,a,f and Namita Roy Choudhury*,a,f

${ }^{a}$ School of Engineering, RMIT University, La Trobe Street, Melbourne, Victoria 3000, Australia.

${ }^{b}$ School of Science, RMIT University, La Trobe Street, Melbourne, Victoria 3000, Australia

${ }^{c}$ Australian Centre for Neutron Scattering, Australian Nuclear Science and Technology

Organisation (ANSTO), New Illawarra Road, Lucas Heights, New South Wales 2234, Australia

${ }^{d}$ Centre of Polymer Systems, Tomas Bata University in Zlin, Nam. T. G. Masaryka 5555, 76001 Zlin, Czech Republic

${ }^{e}$ Faculty of Health and Medical Sciences, The University of Adelaide, North Terrace, Adelaide, SA, 5005, Australia

${ }^{f}$ School of Chemical Engineering and Advanced Materials, The University of Adelaide, North Terrace, Adelaide, SA, 5005, Australia

* Corresponding Author E-mail: namita.choudhury@rmit.edu.au; naba.dutta@rmit.edu.au 


\section{ABSTRACT}

Biomimetic hydrogels offer a new platform for hierarchical structure controlled tough, biocompatible, mechanically tunable and printable gels for regenerative medicine. Herein we report for the first time, the detailed effects of various kinds of nanocellulose namely, bacterial nanocellulose (BC), cellulose nanofibers (CNF) and cellulose nanocrystals (CNC) on the morphology, structure-property relationship and 3D printability of the photochemically crosslinked regenerated silk fibroin (RSF)/nanocellulose composite hydrogels. The hierarchical structure of fabricated biomimetic hydrogels was both qualitatively and quantitatively investigated using scanning electron microscopy and small/ultra-small-angle neutron scattering, whereas their mechanical properties were assessed using rheology, tensile and indentation tests. The micropore size and inter-hydrophobic domain distance of fabricated hydrogels were tuned in the range of 1.8-9.2 $\mu \mathrm{m}$ and 4.5-17.7 $\mathrm{nm}$, respectively. The composite hydrogels exhibit superior viscoelastic, compressive and tensile mechanical properties compared to pristine RSF hydrogel; where the shear storage modulus, compression modulus, young's modulus and tensile toughness were tuned in the range of 0.4-1.4 $\mathrm{MPa}$, 1.3-3.6 $\mathrm{MPa}, 2.2-14.0 \mathrm{MPa}$ and 16.7-108.3 kJ/m³, respectively. Moreover, the obtained mechanical modulus of the composite hydrogels in terms of shear, tensile and compression are comparable to articular cartilage (0.4-1.6 MPa), native femoral artery $(\sim 9.0 \mathrm{MPa})$ and human medial meniscus $(\sim 1.0 \mathrm{MPa})$ tissues, respectively, which demonstrate their potential for a wide range of tissue engineering application. The whisker form of nanocellulose was observed to enhance the printability of composite hydrogels, whereas the fiber form enhanced the overall toughness of the composite hydrogels and promoted the fibroblast cell attachment, viability and proliferation. The results presented here have implications for both fundamental understanding and potential application of RSF/nanocellulose composite hydrogels for 3D printed scaffolds and tissue engineering. 
Keywords: Silk fibroin; Nanocellulose; Composite hydrogel; Hierarchical structure; Mechanical properties; Cellular response; $3 D$ printing

\section{INTRODUCTION}

Three-dimensional (3D) biomimetic hydrogels made from proteins and polysaccharides represent a promising class of biomaterials, which offer a versatile platform for the fabrication of biologically active scaffolds. ${ }^{1-2}$ The building blocks of such biomimetic composites composed of two distinct phases are often arranged in a hierarchical multi-scale manner with functional gradients and heterogeneity. Among proteins and polysaccharides of natural origin, silk and cellulose are the widely used sustainable biomaterials for tissue engineering applications because of their hydrogel forming ability, high mechanical strength and biocompatibility. ${ }^{3-4}$ While silk hydrogels are commonly fabricated from regenerated silk fibroin (RSF) solution, cellulose hydrogels are fabricated from nanocellulose dispersions..$^{3-4}$ We have previously described a facile photochemical crosslinking method, which crosslinks tyrosine amino acid residues in proteins to fabricate RSF and RSF-based hybrid and composite hydrogels. ${ }^{5-8}$ The structural development of photocrosslinked RSF hydrogel from aqueous RSF solution was also reported using small-angle scattering (SAS) experiments, ${ }^{9}$ along with excellent biocompatibility and tunable modulus of a few megapascals. ${ }^{5-7}$ However, compared to RSF fibers, the tissue engineering application of fabricated hydrogels is limited by their brittle mechanical properties, where toughness is required in particular for load-bearing applications. ${ }^{10}$ Conversely, despite many advantages (good mechanical strength), the tissue engineering application of cellulose is often limited by its poor in vivo degradability, non-specific protein binding affinity and poor mammalian cell attachment. ${ }^{11}$ However, the cellular recognition of cellulose has been reported to improve when combined with the fibrous protein. ${ }^{12}$ Therefore, the amalgamation of bioactive RSF and tough 
nanocellulose could potentially overcome their respective material limitations for desired tissue engineering applications. Nanocellulose has been successfully employed as a reinforcing material in many biodegradable polymers and hydrogels. ${ }^{13-15} \mathrm{~A}$ few attempts have been made previously to develop RSF/nanocellulose composites including electrospun nanofibers mats (nanocellulose into RSF matrix), ${ }^{16}$ solution cast films (co-mixed), ${ }^{17}$ and freeze-dried lamellae structures. ${ }^{18}$ However, each study employed different types of nanocellulose with different crosslinking mechanisms which make it difficult to understand the role of cellulose nanostructure in improving the desired properties. Moreover, the composite hydrogels reported so far are only fabricated by physical crosslinking of RSF, where the fabricated structures exhibit either poor water sorption properties and/or low mechanical strength. ${ }^{16-18}$

Herein we present the first report on incorporation of different types of nanocellulose, namely bacterial nanocellulose (BC), cellulose nanofibers (CNF) and cellulose nanocrystals (CNC) into chemically crosslinked RSF hydrogel matrix, and investigation of the influence of morphologically different nanocellulose as reinforcing material at nanoscale on the structure and performance of the composite gels in various aspects. In addition, extrusionbased 3D printing, which enables rapid and customized 3D hydrogel scaffolds has gained increased research attention lately in the field of tissue engineering. ${ }^{19}$ Existing reports on 3D printing of RSF- or nanocellulose-based hydrogels have shown great potential for both systems in tissue engineering applications. ${ }^{20-21}$ Lately, Huang et al. ${ }^{22}$ reported the first example of addition of $\mathrm{BC}$ to improve shape fidelity and mechanical strength of RSF-based composite structures fabricated by 3D printing and lyophilization (physical crosslinking). However, 3D printing of chemically crosslinked RSF/nanocellulose composite gel systems with good resolution and structural integrity remains an experimental challenge and unexplored. Moreover, the printing accuracy or resolution of a composite ink depends on the 
overall viscosity and the interactions between the individual materials which can directly influence the structural recovery. ${ }^{23}$ It is hypothesized that the interaction between $-\mathrm{OH}$ groups of cellulose molecule (C2 and $\mathrm{C} 3$ carbons) and - $\mathrm{NH}$ groups of RSF molecule through strong hydrogen bonding could increase the viscosity and structural recovery to improve the printing resolution. ${ }^{24}$ Therefore, with a view to achieve first chemically crosslinked and structurally stable 3D printable RSF/nanocellulose composite hydrogels, this study examined the crosslinking kinetics and flow properties of RSF/nanocellulose inks using rheology and optimized several printing parameters to understand the printability. Moreover, the effects of different types of nanocellulose on the structure-property relationship, cell viability/proliferation and printing ability of the fabricated RSF/nanocellulose composite hydrogels are presented for potential tissue engineering application.

\section{EXPERIMENTAL SECTION}

\section{Materials}

Silk fibers (from Bombyx mori silk worm) were procured from Beautiful Silks, Australia. CNC (slurry, $12 \mathrm{wt} \%$ solids) and CNF (slurry, $3 \mathrm{wt} \%$ solids) were kindly supplied by Cellulose Lab, Canada. Sodium carbonate $\left(\mathrm{Na}_{2} \mathrm{CO}_{3}\right)$, calcium chloride $\left(\mathrm{CaCl}_{2}\right)$, sodium hydroxide $(\mathrm{NaOH})$ and ethanol were obtained from Chem-Supply, Australia. Deuterium oxide $\left(\mathrm{D}_{2} \mathrm{O}\right)$ was provided by the Australian Nuclear Science and Technology Organisation (ANSTO), Sydney. Tris(2,2-bipyridyl) dichlororuthenium (II) hexahydrate [RuII(bpy3)], phosphate buffered saline (PBS) - pH 7.4, ammonium persulfate (APS) and L929 fibroblast (mouse) cell line were obtained from Sigma-Aldrich, Australia. Dulbecco's Modified Eagle's Medium GlutaMax (DMEM-GX), trypsin, Trypan Blue, fetal bovine serum (FBS) and penicillin-streptomycin (PS) were obtained from Life Technologies, USA. CellTitler $96^{\circledR}$ Aqueous One Solution reagent was obtained from Promega, USA. Live Dead Cell Assay Kit 
was procured from Abcam, UK. Paraformaldehyde and sodium cacodylate buffer were obtained from ProSciTech, Australia.

\section{Preparation of bacterial cellulose $(\mathrm{BC})$}

The $\mathrm{BC}$ was synthesized in the form of pellicle using the bacterial strain Gluconacetobacter xylinus (CCM 3611T) in basal synthetic H-S nutritive medium, incubated for 15 days at 30 ${ }^{\circ} \mathrm{C} .{ }^{25}$ The obtained $\mathrm{BC}$ pellicle was washed with $0.5 \mathrm{~N} \mathrm{NaOH}$ solution (at $80{ }^{\circ} \mathrm{C}$ ) to remove the contaminations, and lyophilized. For BC dispersion preparation, the synthesized BC pellicle was first cut into very small pieces followed by ultrasonication in Milli-Q water (on an ice bath) for 2 hours. The obtained BC in water dispersion was concentrated to $3 \mathrm{wt} \%$ by air-drying.

\section{Silk fibroin solution processing}

Aqueous solution of RSF was prepared following reported protocol..$^{5}$ Briefly, raw Bombyx mori silk fibers were boiled in $0.05 \mathrm{M}$ aqueous $\mathrm{Na}_{2} \mathrm{CO}_{3}$ solution (45 minutes) followed by rinsing several times with water and air-drying. The dried silk fibroin fibers were then dissolved in aqueous solution containing $\mathrm{CaCl}_{2}$ and ethanol (molar ratio of $\mathrm{CaCl}_{2}$ : water: ethanol is $1: 8: 2)$ with fibroin to solvent ratio $(\mathrm{w} / \mathrm{v})$ of $1: 10$. The obtained solution was then filtered (using $1.0 \mu \mathrm{m}$ syringe filter), dialyzed for 4 days against Milli-Q water (using 3.5K MWCO cellulose dialysis tubing), and centrifuged at 10,000 rpm (30 min). The obtained supernatant was air-dried to obtain $\sim 30 \mathrm{wt} \%$ stock solution, which was re-filtered and stored at $4{ }^{\circ} \mathrm{C}$ to prevent gelation and used within a week. The apparent molecular weight of RSF prepared using this method is 37 to $200 \mathrm{kDa}$ determined by Polyacrylamide gel electrophoresis. ${ }^{8}$ 


\section{Hydrogel preparation}

The hydrogels were fabricated by a photocrosslinking method, as reported previously. ${ }^{5}$ Briefly, for pristine RSF hydrogel fabrication, the aqueous RuBPY (photocatalyst) solution was stirred with RSF solution for $20 \mathrm{~min}$, followed by addition and stirring $(5 \mathrm{~min}$ ) of aqueous APS (electron acceptor) solution. The final concentrations of the RSF, APS, and RuBPY were $200 \mathrm{mg} / \mathrm{mL}, 28 \mathrm{mM}$, and $5 \mathrm{mM}$, respectively. The solution mixture (ink) was casted in a Teflon mold (10 $\mathrm{mm}$ diameter and $2 \mathrm{~mm}$ depth), and exposed to a white light source $(50 \mathrm{~W})$ for a period of $2 \mathrm{~min}$. The crosslinked gels were exposed for a further 30 second period by turning the gel upside down to ensure complete cross-linking. A $200 \mathrm{mg} / \mathrm{ml}$ or $20 \mathrm{wt} \%$ silk fibroin composition was used for fabricating photocrosslinked hydrogels as it has been previously reported to be suitable for 3D-printing silk-based hydrogel systems ${ }^{20}$ For the fabrication of RSF/nanocellulose composite hydrogels, nanocellulose dispersions were added to the RSF solution and stirred for 3 hours to make homogeneous mixtures. Then, aqueous RuBPY was added and stirred followed by APS to make the RSF, nanocellulose, APS, and RuBPY final concentrations of $190 \mathrm{mg} / \mathrm{mL}, 10 \mathrm{mg} / \mathrm{mL}, 28 \mathrm{mM}$, and $5 \mathrm{mM}$, respectively. The solution mixtures (inks) were crosslinked as described above. As the CNF and $\mathrm{BC}$ stock used in this work is $3 \mathrm{wt} \%$ solids (highest concentration achievable), their volume of addition into crosslinking ink was limited to a maximum achievable final ink component concentration of $10 \mathrm{mg} / \mathrm{ml}$ nanocellulose. The photocrosslinked solid samples were dialyzed, and equilibration swollen by immersion in water, and subsequently freezedried. The equilibrium water swollen hydrogels were used as such to measure swelling and mechanical properties, whereas the freeze-dried gels were used for other characterization.

\section{X-ray diffraction (XRD)}

The structure of nanocellulose was analyzed using a benchtop MiniFlex X-Ray Diffractometer (Rigaku, Japan). The diffraction patterns were measured at a scan rate of 1.2 
$\circ / \mathrm{min}$ with a step size of $0.02^{\circ}$. The crystallinity index $\left(I_{c}\right)$ of nanocellulose was determined by using the equation $1 .^{26}$

$$
I_{C}=\frac{I_{200}-I_{a m}}{I_{200}} \times 100
$$

where $I_{200}$ is the maximum intensity of crystalline peak $\left(2 \theta=22.5^{\circ}\right)$, and $I_{a m}$ is the maximum intensity of the amorphous peak $\left(2 \theta=18.0^{\circ}\right)$. The mean crystallite size $(\tau)$ of nanocellulose was estimated using the Scherrer's formula (equation 2). ${ }^{27}$

$$
\tau=\frac{K \lambda}{\beta \cos \theta}
$$

where $K$ is the correction factor $(0.94), \lambda$ is the wavelength of X-ray $(0.154 \mathrm{~nm}), \beta$ is the full width half maximum (in radians) of $22.5^{\circ}$ peak and $\theta$ is the angle of diffraction.

\section{Atomic force microscopy (AFM)}

The morphological characterization of nanocellulose was analyzed using a Bruker Dimension Icon AFM equipped with silicon tip on nitride lever (spring constant $(\mathrm{k})=0.4 \mathrm{~N} / \mathrm{m}$, and frequency $\left.\left(f_{0}\right)=70 \mathrm{kHz}\right)$. Samples were drop casted on silicon substrate and air-dried for analysis. Measurements were performed in ScanAsyst - AIR mode, and the images were analyzed using the Gwyddion software. The dimensions of nanocellulose were obtained using the ImageJ software.

\section{Water uptake studies}

The equilibrium water uptake capacity $\left(\mathrm{C}_{\mathrm{w}}\right)$ of the fabricated hydrogels were calculated using equation $3 .^{28}$ The equilibrium water swollen weight $\left(W_{s}\right)$ of fabricated hydrogels (after the excess water on the surface was removed using blotting paper) was measured after 2 days of dialysis as it require 25 hours to completely rehydrate (based on our previously reported work). ${ }^{5}$ The weighed samples were air- and vacuum-dried, respectively to determine the dry 
weight $\left(W_{d}\right)$. Each experiment is repeated thrice, and experimental results were presented as mean values with standard deviation

$$
C_{w}=\frac{W_{s}-W_{d}}{W_{d}}
$$

\section{Scanning electron microscopy (SEM)}

Philips XL30 Field Emission scanning electron microscope was used to investigate the micropore size and structure of freeze-dried hydrogels and morphology of cultured cells. The freeze-dried samples were cross sectioned, fixed on the SEM stubs and sputter coated with platinum for analysis. The cell culture samples were washed thrice with the complete media and once with sodium cacodylate buffer followed by fixing them with $100 \mu \mathrm{L}$ of primary fixative (glutaraldehyde/paraformaldehyde) for $30 \mathrm{~min}$ and rinsing them thrice with sodium cacodylate buffer for 5 minutes each. The secondary fixation was performed using $100 \mu \mathrm{l}$ of $1 \%$ osmium tetroxide for 30 minutes in dark condition, and the samples were then washed with milli-Q water and successively passed through 50\%, 70\%, 90\%, 95\% and $100 \%$ ethanol (10 min for each step) and air-dried overnight. All samples were fixed on SEM stubs and sputter coated with platinum for analysis.

\section{Small-angle neutron scattering (SANS)}

The structure and morphology of nanocellulose dispersed in $\mathrm{D}_{2} \mathrm{O}$, and fabricated hydrogels equilibrium swollen in $\mathrm{D}_{2} \mathrm{O}$ were analyzed using the Quokka SANS instrument at ANSTO. ${ }^{29}$ The nanocellulose were repeatedly dialyzed against $\mathrm{D}_{2} \mathrm{O}$ and loaded into a $2 \mathrm{~mm}$ path length Quokka cell assembled with $20 \mathrm{~mm}$ diameter quartz windows. The $\mathrm{D}_{2} \mathrm{O}$ swollen hydrogels were loaded into the same demountable Quokka cell assembly, along with excess $\mathrm{D}_{2} \mathrm{O}$. The scattering data of samples (at $25^{\circ} \mathrm{C}$ ) were collected in the scattering vector, $q$ (equation 4$)^{30}$ range of $0.001-0.3 \AA^{-1}$ using a $12.5 \mathrm{~mm}$ (diameter) sample aperture, sample to source 
aperture distances of 20,12 and $2 \mathrm{~m}$, and a neutron wavelength $(\lambda)$ of $8.1 \AA^{-1}$ (for lens optics) and $5 \AA^{-1}$, respectively.

$$
q=\frac{4 \pi \sin \theta}{\lambda}
$$

where $2 \theta$ is the angle of scattering. The high neutron $\operatorname{SLD}\left(6.36 \times 10^{-6} \AA^{-2}\right)$ of $\mathrm{D}_{2} \mathrm{O}$ provide required contrast against cellulose and protein, and also reduce any incoherent background scattering. ${ }^{31}$ The obtained scattering data were processed and converted to absolute scale using the IgorPro software package (loaded with NCNR SANS reduction macros) ${ }^{32}$ The PRIMUS computer program was used to subtract $\mathrm{D}_{2} \mathrm{O}$ background scattering from the measured sample data. ${ }^{33}$ Furthermore, the SasView computer program was used to determine incoherent background scattering values (through a high- $q$ power law fit), which were later subtracted from the respective sample data for analysis. ${ }^{34}$ The structural parameters of cellulose dispersions and fabricated hydrogels were obtained using the SasView computer program through appropriate model functions fits to scattering data.

\section{Ultra-small-angle neutron scattering (USANS)}

The hierarchical structure of fabricated hydrogels equilibrium swollen in $\mathrm{D}_{2} \mathrm{O}$ were analyzed using the Kookaburra USANS instrument at ANSTO. ${ }^{35}$ The same Quokka cells used for SANS experiments were used to measure USANS data. The sample data (measured at room temperature) were collected in the $q$-range $0.00006-0.001 \AA^{-1}$ using a $12.5 \mathrm{~mm}$ sample aperture diameter and a neutron wavelength of $4.74 \AA$. The measured scattering data were processed in Gumtree (using Python scripts written for Kookaburra) and de-smeared using the Lake algorithm. ${ }^{36}$ The structural parameters of the fabricated hydrogels were obtained using the SasView computer program through appropriate model functions fits to scattering data. 


\section{Fourier transform infrared (FTIR) spectroscopy}

Nicolet 6700 FTIR spectrometer (Thermo Fisher Scientific, Australia) equipped with Attenuated Total Reflection (ATR) was used to analyze the secondary structure of the freezedried hydrogels. The data was measured in the wavelength range of $900-4000 \mathrm{~cm}^{-1}$ and processed through OMNIC software.

\section{Rheology}

The viscoelastic properties of equilibrium water swollen hydrogels and flow properties of RSF/nanocellulose inks (measured under dark environment) were analyzed using Discovery hybrid rheometer (TA instruments, Delaware, USA) operated at room temperature. Viscoelastic properties of the hydrogels were investigated in oscillatory shear mode using 8 mm parallel plate geometry under $10 \%$ compression. The mechanical spectra were collected over a strain $\%$ of 0.01 to 50 at a fixed frequency $(1 \mathrm{rad} / \mathrm{s})$. The dynamic frequency sweep was conducted in the linear regime $(0.05 \%$ strain $)$ over the frequency range of $0.01-10 \mathrm{rad} / \mathrm{s}$. Rotational rheology was used to study the flow properties of the hybrid inks using a cone and plate geometry of $20 \mathrm{~mm}$ diameter with a cone angle of $1^{\circ}$ at a constant gap of $0.053 \mathrm{~mm}$ by varying the shear rates from $0.02 \mathrm{~s}^{-1}$ to $10 \mathrm{~s}^{-1}$. The structural (viscosity) recovery properties were analyzed in rotational mode by varying the shear rates from $0.1 \mathrm{~s}^{-1}$ to $90 \mathrm{~s}^{-1}$ followed by $0.1 \mathrm{~s}^{-1}$. All the data was collected and processed using the TRIOS software. The photorheology of pristine RSF and RSF/nanocellulose inks at $1 \mathrm{rad} / \mathrm{s}$ and 0.1 strain \% was performed in oscillatory mode using a MCR-301 Anton Paar rheometer (Anton Paar, GmbH) operated at room temperature. The experiment was performed with sample loaded between transparent quartz plate, and the $10 \mathrm{~mm}$ cone plate with a cone angle of $1^{\circ}$ at constant gap of $0.031 \mathrm{~mm}$. Photocuring light of $460 \mathrm{~nm}$ wavelength and $10 \mathrm{~mW}$ intensity was turned on at 100 second during the experiment. 


\section{Tensile testing}

The tensile mechanical properties of equilibrium water swollen hydrogels were assessed using an Intron 4465 tensiometer equipped with $2 \mathrm{kN}$ load cell at room temperature. The rectangular hydrogel samples with dimensions $30 \mathrm{~mm}$ length, $10 \mathrm{~mm}$ width and $2 \mathrm{~mm}$ thickness were used for tensile experiment with a crosshead speed of $10 \mathrm{~mm} / \mathrm{min}$. The data was processed using the BlueHill software and the toughness of the hydrogels was calculated by integrating the area under the stress-strain curve.

\section{Microindentation}

The compressive mechanical properties of equilibrium water swollen hydrogels were measured using a Bose Electroforce 5500 mechanical tester equipped with $250 \mathrm{~g}$ load cell and a cylindrical probe of $1 \mathrm{~mm}$ diameter. The measurements (up to $20 \%$ compression) were performed on cylindrical gels of $10 \mathrm{~mm}$ diameter and $\sim 2 \mathrm{~mm}$ thickness with a constant probe displacement rate of $0.1 \mathrm{~mm} / \mathrm{sec}$. The measured force was normalized by the probes crosssectional area to obtain stress; whereas strain was calculated from the displacement of the probe.

\section{In vitro cell attachment, viability and proliferation}

The L929 fibroblast cell line was sub-cultured in DMEM-GX media boosted with $10 \%$ FBS and $1 \%$ PS. The cells were incubated at $37{ }^{\circ} \mathrm{C}$ in a $5 \% \mathrm{CO}_{2}$ atmosphere and trypsinized prior to passaging. The subcultured cells were used at passage 9 after defrosting. A total of 4.6 million cells per ml were achieved with the cell viability of $94 \%$ when counted using Trypan Blue assay. For proliferation experiment, the hydrogels were fabricated directly in a 96-well plate using $100 \mu \mathrm{L}$ of crosslinking solution, dialyzed against milli-Q water, sterilized with $80 \%$ alcohol for 4 hours, and subsequently soaked in cell culture medium for 2 days to ensure it is completely rehydrated. The L929 cells were dropped on the hydrogel samples at a 
density of $1 \times 10^{4}$ cells/well, topped up with $100 \mu \mathrm{l}$ media, and placed in an incubator (in the presence of $5 \% \mathrm{CO}_{2}$ at $37^{\circ} \mathrm{C}$ ) for 5 days. The cell viability of hydrogels was also quantitatively assessed on days 1, 3 and 5 using Trypan Blue dye. For this, $10 \mu$ of trypsinized cells was mixed with $10 \mu \mathrm{l}$ of Trypan Blue and assessed using Invitrogen Countess Automated Cell Counter. The proliferation of cells on hydrogels was evaluated on days 1, 3 and 5 using the MTS assay. The tetrazolium compound [3-(4,5-dimethyl-2-yl)-5-(3carboxymethoxyphenyl)-2-(4-sulfophenyl)-2H-tetrazolium (MTS) converts to formazan product in the reducing environment of living cells, which can be quantitatively measured. For this, $10 \mu \mathrm{l}$ of MTS was added to each plate and subjected to incubation for 4 hours. The formazan supernatant is transferred to a new 96-well plate and read immediately using a microplate reader (SpectraMax) at an absorption wavelength of $490 \mathrm{~nm}$. Cell proliferation on tissue culture plastic (TCP) was used as a positive control group. For SEM imaging, the cells were cultured in a 12 well plate containing $500 \mu \mathrm{L}$ of cured gel at a density of $200 \mathrm{~K}$ cells per each well, and the morphology was evaluated on days 1, 3 and 5 after fixing them using primary and secondary fixative (details of fixatives are shown in SEM sample preparation).

\section{D printing}

The pristine RSF and RSF/nanocellulose composite hydrogels were 3D printed from photocrosslinkable precursor solutions (inks) using an extrusion based GeSiM BioScaffolder printer operated through the GeSIM Robotics software. For this, $3 \mathrm{~mL}$ of prepared ink was loaded into a $10 \mathrm{~mL}$ cartridge, extruded through a nozzle of diameter 23 Gauge, and printed on a glass substrate, with a start and end break of $0.1 \mathrm{sec}$. The layer by layer strands were printed with a speed of $20 \mathrm{~mm} / \mathrm{s}$ at varying pressure between $20-35 \mathrm{kPa}$. The strand height and strand width were chosen to be $0.1 \mathrm{~mm}$ and $0.58 \mathrm{~mm}$, respectively, with a Z-offset action of 0.1-0.2 mm. The printed inks were photo-cured for each layer using white light for 40 seconds to obtain a chemically crosslinked stable structure. The dimensions of the printed 
constructs were measured using the ImageJ software and the printing accuracy was calculated using the method reported previously. ${ }^{37}$ The printing accuracy $(P)$ of the system was calculated (as average from 4 measurements) from the printed width $\left(W_{i}\right)$ and experimental width $(W)$ using the equation 5 .

$$
P=\left(1-\frac{W_{i}-W}{W}\right) * 100
$$

\section{RESULTS AND DISCUSSION}

\section{Structure and morphology of the different types of nanocellulose used}

The structure and morphology of $\mathrm{CNC}, \mathrm{CNF}$ and $\mathrm{BC}$ were investigated using $\mathrm{XRD}, \mathrm{AFM}$ and SANS analysis (see Supporting Information). The CNC exhibited XRD peaks of both cellulose I and cellulose II structures, whereas the CNF and BC exhibited only cellulose I structure (Figure S1 (A) in Supporting Information). ${ }^{38}$ A cellulose I/cellulose II ratio of $\sim 4.2$ was estimated for the CNC by deconvoluting the XRD data (Figure S1 (B) in Supporting Information). The crystallinity index of $\mathrm{CNC}, \mathrm{CNF}$ and $\mathrm{BC}$ was estimated as $\sim 91.9 \%$, $\sim 79.8 \%$ and $\sim 96.3 \%$, respectively. Moreover, the mean crystallite sizes of CNC, CNF and BC was estimated as $\sim 4.1 \mathrm{~nm}, \sim 4.5 \mathrm{~nm}$ and $\sim 5.6 \mathrm{~nm}$, respectively. Figure $\mathrm{S} 1$ (C) in Supporting Information show the $\mathrm{AFM}$ images of $\mathrm{CNC}, \mathrm{CNF}$ and $\mathrm{BC}$, respectively; where the nanocelluloses show a tendency to form bundles along their long axis. The CNC exhibited a needle-like morphology with fibrillar to bundle diameter in the range of 5-20 nm and length (polydispersed) in the range of 100-400 nm, respectively. The CNF and $\mathrm{BC}$ exhibited several micrometers long (higher aspect ratio) fiber-like and ribbon-like morphology, respectively with fibrillar/ribbon to bundle diameter/width in the range of 5-30 nm and 10-40 nm, respectively. Conversely, Figure S2 in Supporting Information shows the SANS data of water swollen nanocelluloses and their model function fits. The diameter (d) or width of water 
swollen $\mathrm{CNC}, \mathrm{CNF}$ and $\mathrm{BC}$ fibrils was estimated (from the $q$-limit using the relation $\mathrm{d}=$ $2 \pi / \mathrm{q})^{9}$ to be $\sim 9.0 \mathrm{~nm}, \sim 7.0 \mathrm{~nm}$ and $\sim 44.8 \mathrm{~nm}$, respectively. Therefore, from XRD, AFM and SANS results, it is established that the $\mathrm{CNC}$ and $\mathrm{CNF}$ occur largely as fibrils and the $\mathrm{BC}$ as bundles; where the concentration fluctuations (or hierarchical amorphousness) in nanocellulose fibrils/ribbons (from high- $q$ SANS slope) was observed in the order of BC > CNF > CNC (see Supporting Information for details). Therefore, for a given mass, the surface area of nanocellulose used would be in the order of CNC $>$ CNF $>$ BC. The observed differences in structure, crystallinity, morphology, size, aspect ratio and surface area of nanocellulose used are hypothesized to enable a wide range of specific hierarchical structure and physicochemical properties in the fabricated composite hydrogels. ${ }^{14-15}$

\section{Equilibrium water swelling and microstructure of fabricated hydrogels}

For hydrogels to be applied as tissue engineering scaffolds, the water uptake capacity $\left(\mathrm{C}_{\mathrm{w}}\right)$ and microstructure are quite important properties to consider as it influences the viscoelastic property and cell-hydrogel interactions. ${ }^{39}$ Therefore, the effect of different types of nanocellulose on the water swelling property of the fabricated RSF/nanocellulose composite hydrogels was characterized both qualitatively and quantitatively. Figure 1A shows the optical images of the fabricated hydrogels at equilibrium swollen state. The optical size or diameter of the RSF hydrogel was observed to decrease with the incorporation of nanocellulose. The optical size of equilibrium swollen hydrogels were observed in the order of $\mathrm{RSF}>\mathrm{RSF} / \mathrm{BC}>\mathrm{RSF} / \mathrm{CNF}>\mathrm{RSF} / \mathrm{CNC}$. The calculated $\mathrm{C}_{\mathrm{w}}$ of the fabricated hydrogels is presented in Table 1. The pristine RSF hydrogel exhibited a $C_{w}$ value of $13.8 \pm 0.6$, which decreased with the incorporation of nanocellulose. The fabricated hydrogels exhibited $\mathrm{C}_{\mathrm{w}}$ in the order of $\mathrm{RSF}>\mathrm{RSF} / \mathrm{BC}>\mathrm{RSF} / \mathrm{CNF}>\mathrm{RSF} / \mathrm{CNC}$, which is in general agreement with the observed optical size trend. A similar trend in reduction of swelling capacity with the incorporation of CNC has also been reported for gelatin hydrogels. ${ }^{40}$ 
Table 1. Water uptake results and estimated structural parameters of fabricated hydrogels.

\begin{tabular}{|c|c|c|c|c|c|}
\hline \multirow[t]{3}{*}{ Sample } & \multirow{3}{*}{$\begin{array}{c}\text { Swelling } \\
\text { Water } \\
\text { uptake } \\
\text { capacity } \\
\left(C_{w}\right)\end{array}$} & \multirow{3}{*}{$\begin{array}{c}\text { SEM } \\
\text { Micropore } \\
\text { size }(\mu \mathrm{m})\end{array}$} & \multicolumn{3}{|c|}{ a SANS } \\
\hline & & & High-q & Mid- $q$ & Low-q \\
\hline & & & $\begin{array}{c}\text { R } \mathbf{R}_{\mathrm{g}} \text { of } \\
\text { hydrophobic } \\
\text { domain (nm) }\end{array}$ & $\begin{array}{c}\text { Inter- } \\
\text { hydrophobic } \\
\text { domain distance } \\
\text { (nm) }\end{array}$ & $\begin{array}{c}\text { Mass fractal } \\
\text { dimension }\end{array}$ \\
\hline RSF & $13.8 \pm 0.6$ & $9.2 \pm 3.8$ & $2.1 \pm 0.1$ & $15.4 \pm 2.3$ & $2.71 \pm 0.02$ \\
\hline $\mathrm{RSF} / \mathrm{CNC}$ & $7.7 \pm 0.2$ & $1.8 \pm 0.5$ & $2.1 \pm 0.1$ & $5.0 \pm 0.5$ & $2.94 \pm 0.05$ \\
\hline $\mathrm{RSF} / \mathrm{CNF}$ & $11.0 \pm 0.3$ & $5.7 \pm 1.6$ & $2.1 \pm 0.1$ & $8.0 \pm 1.0$ & $2.88 \pm 0.03$ \\
\hline $\mathrm{RSF} / \mathrm{BC}$ & $12.2 \pm 0.4$ & $6.9 \pm 1.7$ & $2.2 \pm 0.2$ & $12.0 \pm 2.0$ & $2.85 \pm 0.02$ \\
\hline
\end{tabular}

${ }^{a}$ Standard deviation values were attained from respective model function fits.

Figure 1B shows the SEM images of fabricated, equilibrium swollen and freeze-dried hydrogels with microporous interconnected structures, which is comparable to that of freezedried RSF/cellulose gels. ${ }^{41}$ The mean pore size values estimated (using the ImageJ software) from SEM images of the fabricated hydrogels are provided in Table 1. The pristine RSF hydrogel showed an average pore size of $9.2 \pm 3.8 \mu \mathrm{m}$, which decreased with the incorporation of nanocellulose. Among the three types of nanocellulose used, CNC was observed to affect the micropore size and structure of RSF hydrogel the most, followed by $\mathrm{CNF}$ and $\mathrm{BC}$, respectively, which agrees with the water uptake results. The decrease in water uptake capacity and micropore size of RSF hydrogel with incorporation of nanocellulose can be attributed to strong inter-molecular interaction, i.e. hydrogen bonding between the $\mathrm{C} 2 / \mathrm{C} 3$ carbon of cellulose and the $-\mathrm{NH}$ group of RSF chain, where the surface area of nanocellulose is observed in the order of $\mathrm{CNC}>\mathrm{CNF}>\mathrm{BC} .^{24}$ Therefore, it is hypothesized that the relatively low $\mathrm{RSF} / \mathrm{CNC}$ composite hydrogel swelling may be due to the relatively large surface area of $\mathrm{CNC}$, where a large number of RSF-CNC hydrogen bond may restrict RSF 
chain movement during swelling leading to smaller pore size. ${ }^{14-15}$ Although both CNF and $\mathrm{BC}$ exhibit higher aspect ratio, the surface area of $\mathrm{CNF}>\mathrm{BC}$; therefore, $\mathrm{RSF} / \mathrm{CNF}$ composite hydrogel shows relatively lower water uptake capacity and smaller pore size compared to the RSF/BC composite hydrogel. Furthermore, the percentage of crystallinity and hierarchical amorphousness in nanocellulose fibrils/ribbons may also influence the equilibrium swelling characteristics of the fabricated hydrogels. ${ }^{14-15}$ Therefore, for a given mass of nanocellulose, the water swelling capacity, micropore size and structure of RSF/nanocellulose composite hydrogels can be tuned based on the type of nanocellulose used as filler. The water swelling is also anticipated to affect the hierarchical structure of fabricated hydrogels. ${ }^{9}$
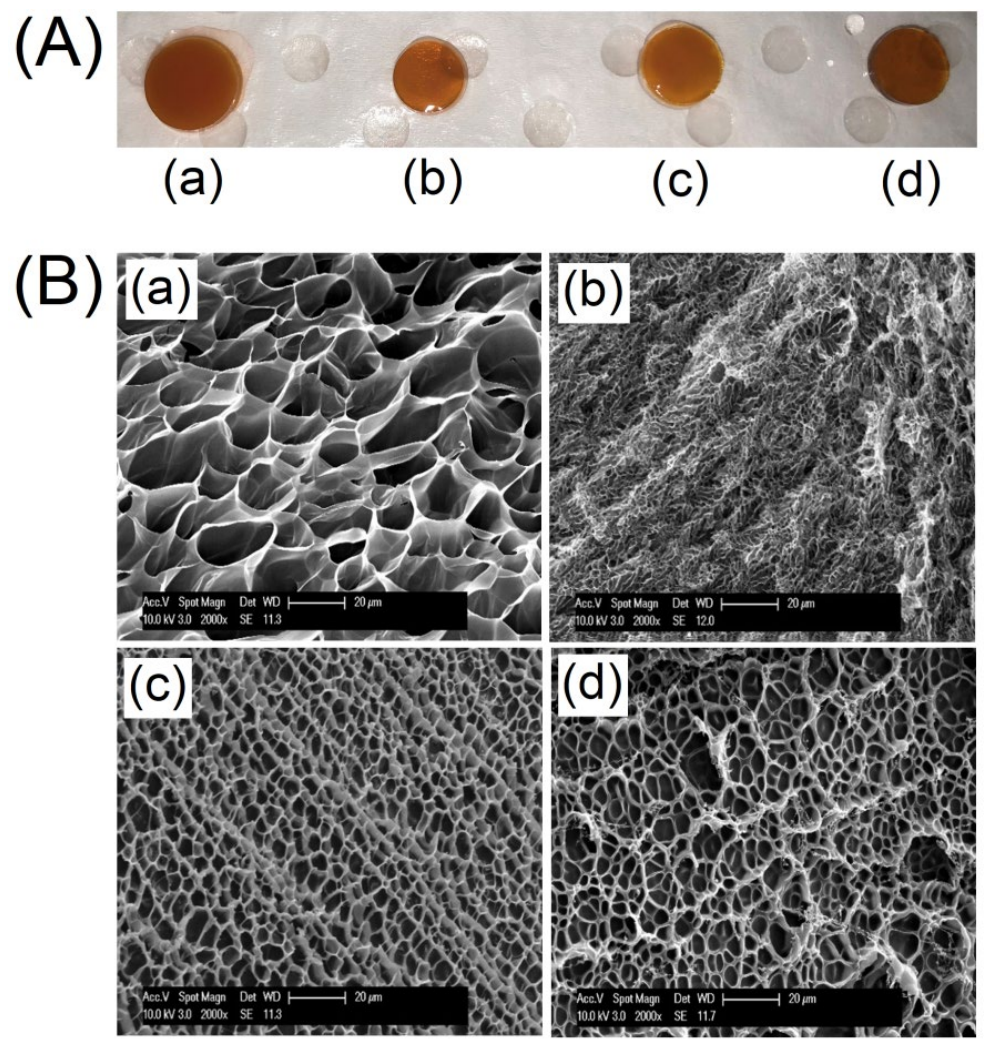

Figure 1. (A) Optical pictures and (B) SEM micrographs (equilibrium water swollen and freeze dried) of fabricated hydrogels; where (a) is RSF hydrogel, (b) is RSF/CNC composite hydrogel, (c) is RSF/CNF composite hydrogel and (d) is RSF/BC composite hydrogel. SEM scale bar is $20 \mu \mathrm{m}$. 


\section{Hierarchical structure of fabricated hydrogels}

In general, the hierarchical structure of a biomimetic hydrogel largely influences its mechanical properties, where the molecular building blocks or domains of the hydrogel transmit properties across the length scales. Therefore, a fundamental knowledge of the hierarchical structure of fabricated hydrogels is crucial for tailoring their mechanical properties for desired applications in tissue engineering. SANS is a powerful experimental technique for analysis of nanostructure and organization of proteins in solution and gel state, and is complementary to electron microscopy. ${ }^{42-43}$ Conversely, USANS offer structural information of gels in microscale. ${ }^{44}$ Therefore, combining SANS and USANS, the effect of incorporating different types of nanocellulose on the hierarchical gel structure of fabricated hydrogels, from $2 \mathrm{~nm}$ to $12.5 \mu \mathrm{m}$, was analyzed both qualitatively and quantitatively. The equilibrium $\mathrm{D}_{2} \mathrm{O}$ swollen pristine RSF hydrogel had a neutron SLD of $\sim 3.2 \times 10^{-6} \AA^{-2}{ }^{8}$ The neutron SLD of $\mathrm{D}_{2} \mathrm{O}$ swollen nanocellulose was calculated as $\sim 2.8 \times 10^{-6} \AA^{-2}$ using the NIST neutron activation and scattering calculator; where the ${ }^{1} \mathrm{HO}(6)$ and ${ }^{1} \mathrm{HO}(2)$ groups of nanocellulose exchange hydrogen with deuterium in $\mathrm{D}_{2} \mathrm{O} .{ }^{45}$ As the neutron SLD of RSF and nanocellulose in fabricated hydrogels are close to each other, it is practically challenging to study their individual structure in the hydrogel matrix. Therefore, the SANS intensity profile of all three nanocellulose samples (for a known concentration of $1 \mathrm{wt} \%$, same as used in hydrogel) in $\mathrm{D}_{2} \mathrm{O}$ were first fitted with model functions (see text and Figure $\mathrm{S} 2$ in Supporting Information), and the fit parameters were used to help with the deconvolution of fabricated composite hydrogel SANS data. The overall neutron scattering intensity, $\mathrm{I}(q)$ of a gel is a function of both the intra-molecular form factor, $\mathrm{P}(q)$, and the inter-molecular structure factor, $\mathrm{S}(q)$, as given in equation $6{ }^{46}$

$$
I(q)=\varphi P(q) S(q) \text {------ }(6)
$$


where $\varphi$ is a scaling factor. The form factor provides information on morphology and size of a molecule/particle (related to the distribution of inter-atomic distances), whereas the structure factor provides information on correlation distances and/or spatial distribution (related to the inter-particle interactions). ${ }^{46}$
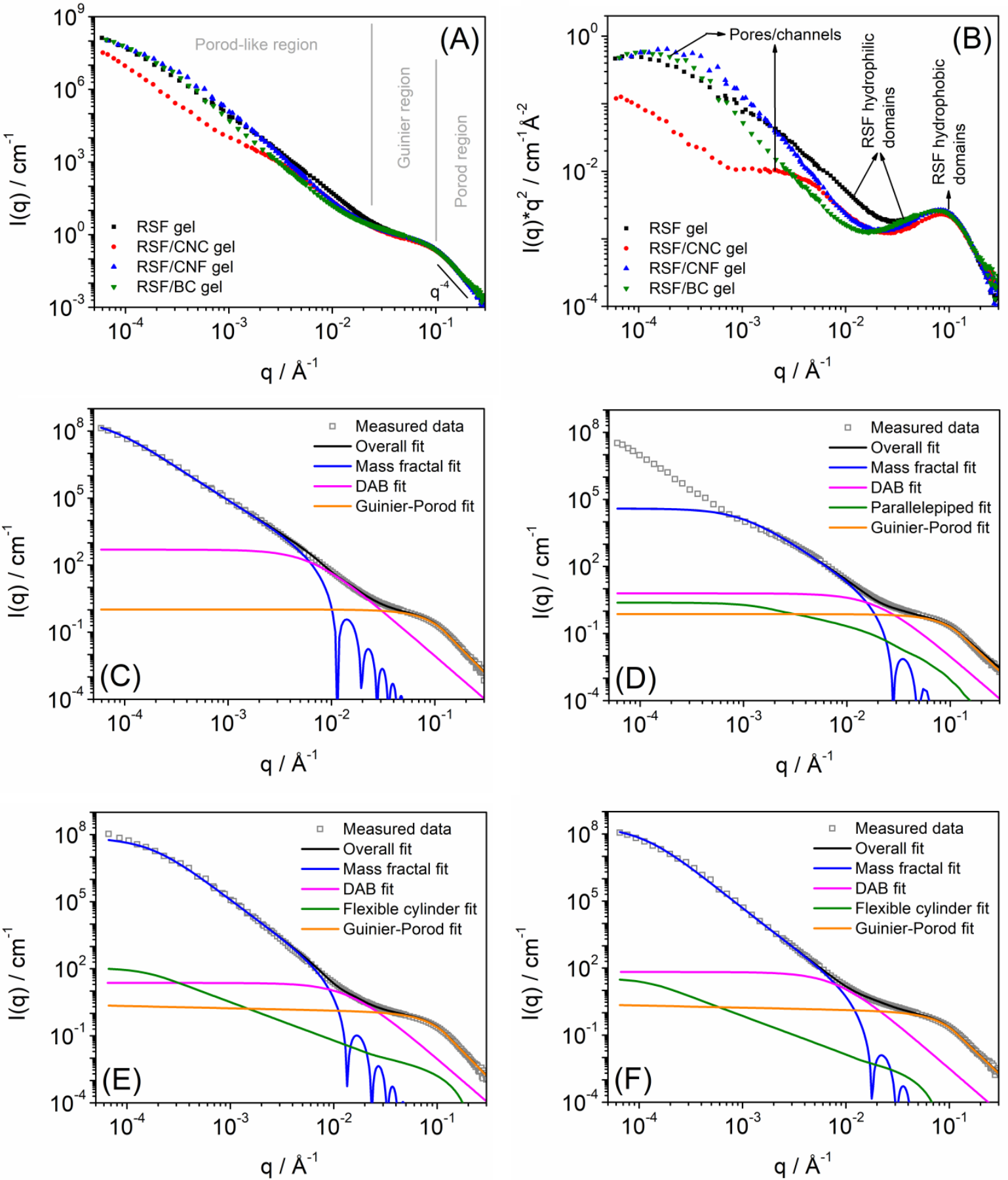
Figure 2. (A) SANS intensity profile and (B) Kratky plot of equilibrium $\mathrm{D}_{2} \mathrm{O}$ swollen fabricated hydrogels. Model function fits to SANS data of (C) RSF hydrogel, (D) RSF/CNC composite hydrogel, (E) RSF/CNF composite hydrogel and (F) RSF/BC composite hydrogel.

The combined SANS and USANS intensity profile of fabricated hydrogels (Figure 2A) at equilibrium swollen state showed a Porod region at high- $q\left(0.1<q<0.3 \AA^{-1}\right)$, a Guinier region at mid- $q\left(0.02<q<0.1 \AA^{-1}\right)$, and a Porod-like region at low- $q(0.00005<q<$ $0.02 \AA^{-1}$ ). A power law fit in the high- $q$ region returned a Porod slope of $\sim 4.0$ (characteristic of a sharp interface) for all fabricated hydrogels, which can be ascribed to hydrophobic $\beta$ sheet domains of RSF (formed by photocrosslinking). ${ }^{9}$ Conversely, the mid- $q$ scattering region is contributed to hydrophilic random coil domains. At the low- $q$ USANS or Porod-like region, the curves show a strong upturn with a slope of $\sim 2.8$ (characteristic of mass fractal structures), which describes the gel-network structure extending beyond the USANS length scales. The broad feature observed on top of this steep upturn can be attributed to micropores and channels of the hydrogels, as observed in SEM. ${ }^{9}$ The observed trends in neutron scattering intensity can be clearly seen from the Kratky plot $\left[\mathrm{I}(q)^{*} q^{2}\right.$ vs. $\left.q\right]$ (Figure $\left.2 \mathrm{~B}\right)$. In order to obtain the structural parameters of fabricated hydrogels, a combined form factor model function was used to fit the scattering data, as given in equation 7 :

$$
I(q)=A(q)+B(q)+C(q)+D(q)
$$

where $\mathrm{A}(q)$ is the mass fractal model, $\mathrm{B}(q)$ is the Debye Anderson Brumberger (DAB) model, $\mathrm{C}(\mathrm{q})$ is the combined form factor model function used for respective nanocellulose data fit (details provided in Supporting Information), and $\mathrm{D}(q)$ is the Guinier-Porod model. The nanocellulose fit parameters were used for $\mathrm{C}(q)$ fitting. The Guinier-Porod model estimates the size and structure of scattering objects, such as RSF hydrophobic domains. ${ }^{47}$ Conversely, the DAB model estimates the correlation length (L) of a randomly distributed two-phase 
system, such as inter-distance between RSF hydrophobic domains. ${ }^{48}$ The mass fractal model estimates the dimension of fractal-like aggregates, such as structural inhomogeneities and/or micropores/channels, where higher values correspond to denser structures. ${ }^{49}$ The obtained structural parameters from model fits (Figure 2C to 2F) are given in Table 1. No change in RSF hydrophobic domain size was observed with nanocellulose incorporation, whereas a significant change in hydrophilic domain size or inter-hydrophobic domain distance was observed. The inter-hydrophobic domain distance was observed to be the largest for pristine RSF hydrogel, and to decrease with different nanocellulose incorporation. The interhydrophobic domain distance of RSF/nanocellulose composite hydrogels were obtained in the order of $\mathrm{RSF} / \mathrm{BC}>\mathrm{RSF} / \mathrm{CNF}>\mathrm{RSF} / \mathrm{CNC}$ hydrogel, which may be related to the intermolecular interactions between RSF and nanocellulose, ${ }^{24}$ and is in common agreement with the water uptake results. As the low- $q$ scattering does not reach a clear Guinier region, the absolute pore size values obtained from the mass fractal fit (not given) needs to be treated with care; however, the obtained fractal dimension is valid (Table 1) and are in common agreement with the SEM results. Moreover, the variations observed in water uptake results of the hydrogels are largely attributed to the difference in micropore/channel size and interhydrophobic domain distance. Therefore, it is conclusive that the incorporation of nanocellulose greatly affects the hierarchical structure at both the nano- and micro-scale, which could greatly influence other physiochemical properties.

\section{Secondary structure of fabricated hydrogels}

The secondary structural differences in the RSF/nanocellulose composite hydrogels with respect to different types of cellulose were estimated both quantitatively and qualitatively using FTIR spectroscopy. Figure 3A compares the FTIR spectra of RSF/nanocellulose composite hydrogels to pristine RSF hydrogel. The spectra of fabricated hydrogels exhibited two major peaks between $1500-1600 \mathrm{~cm}^{-1}$ and $1600-1700 \mathrm{~cm}^{-1}$, related to amide II and amide 
I conformational bands of the protein. ${ }^{50}$ The peak around $1230 \mathrm{~cm}^{-1}$ can be ascribed to the crystalline $\beta$-sheet structure of the RSF. ${ }^{41}$ The secondary structure of hydrogels was quantitatively estimated by deconvoluting the amide I band (Figure S3 in Supporting Information) using frequency values corresponding to $\alpha$-helix $\left(1658 \mathrm{~cm}^{-1}\right)$, aggregate $\beta$ strand $\left(1614 \mathrm{~cm}^{-1}\right)$, intramolecular $\beta$-sheets $\left(1626 \mathrm{~cm}^{-1}\right)$, intermolecular $\beta$-sheet $\left(1697 \mathrm{~cm}^{-1}\right)$, turns $\left(1670 \mathrm{~cm}^{-1}, 1681 \mathrm{~cm}^{-1}\right)$ and random coil $\left(1638 \mathrm{~cm}^{-1}, 1647 \mathrm{~cm}^{-1}, 1652 \mathrm{~cm}^{-1}\right) .{ }^{51-53}$ The evaluated secondary structural contents of the fabricated hydrogels are presented in Figure 3B. It was clearly noticed that the secondary structure of the fabricated hydrogels was dominated by random coil structure, and the incorporation of $\mathrm{CNC}, \mathrm{CNF}$ and $\mathrm{BC}$ increased the total $\beta$-sheet (intra- \& intermolecular interactions) structures in the RSF/nanocellulose composite hydrogels. A similar trend in the $\beta$-sheet content of silk/cellulose films has been previously reported. ${ }^{24}$ The order of total $\beta$-sheet crystal structures resulting from both interand intra-molecular interactions in RSF/nanocellulose composite hydrogels is RSF/BC > $\mathrm{RSF} / \mathrm{CNF}>\mathrm{RSF} / \mathrm{CNC}$. The increase in $\beta$-sheet content, suggests that the nanocellulose strongly interacts with the RSF by hydrogen bonding thereby promoting $\beta$-sheet content, and which could influence the mechanical properties of RSF/nanocellulose composite hydrogels. ${ }^{24,41}$
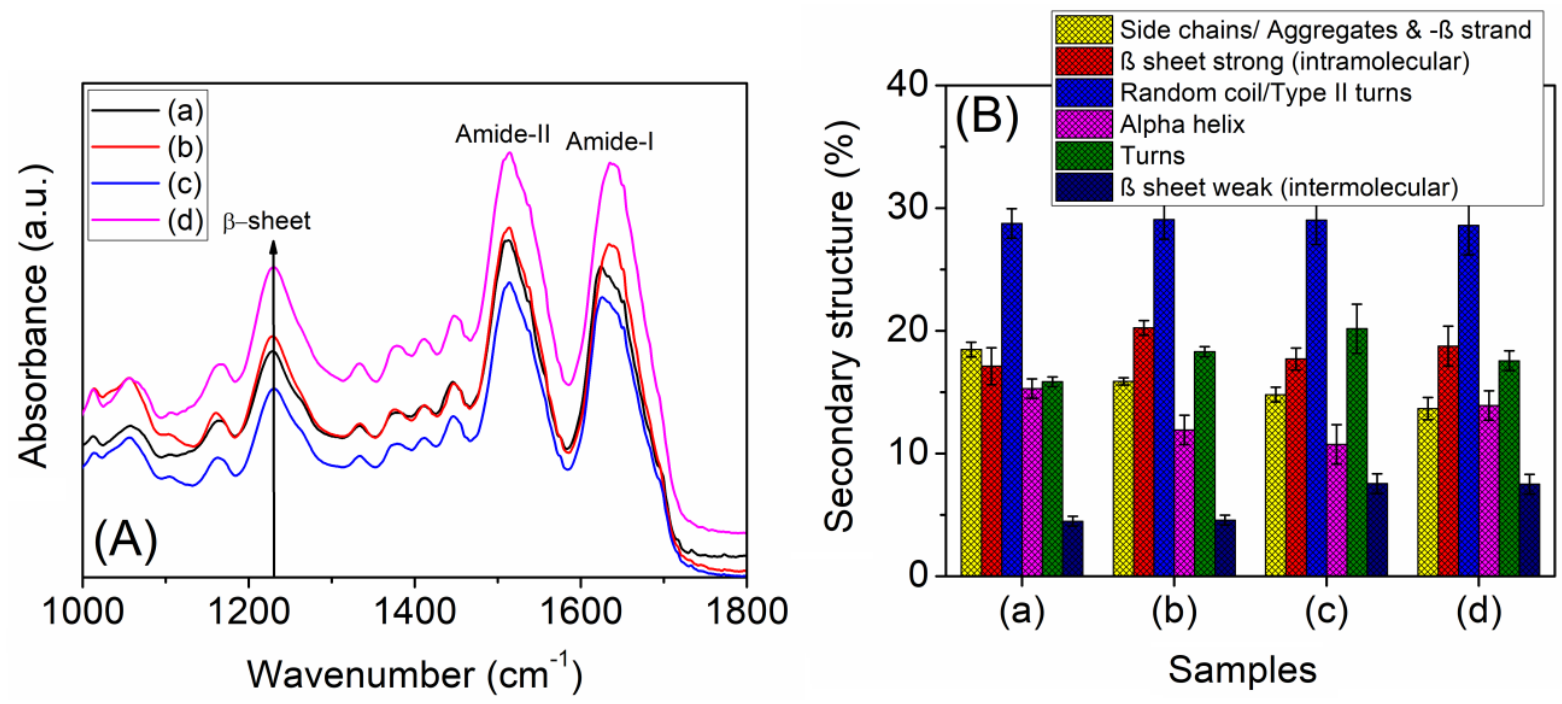
Figure 3. (A) FTIR spectra and (B) quantitative secondary structure estimates from FTIR spectra. The sample codes are (a) RSF hydrogel, (b) RSF/CNC composite hydrogel, (c) $\mathrm{RSF} / \mathrm{CNF}$ composite hydrogel and (d) RSF/BC composite hydrogel.

\section{Viscoelastic mechanical properties of fabricated hydrogels}

Viscoelasticity is an important property of biological tissues and is one of the key parameters to be addressed in the application of scaffolds in biomedicine. The influence of different types of nanocellulose on the viscoelastic behavior of the fabricated RSF/nanocellulose composite hydrogels was investigated using dynamic oscillation shear rheology. It is evident that cellulose from different sources produce nanoparticles with different dimensions and mechanical properties. In general, hydrogels exhibit complex viscoelastic behavior, which can be explained through their experimentally measured storage and loss modulus values. ${ }^{54}$ Figure 4A shows the shear storage modulus (G') and loss modulus (G') trend of the fabricated hydrogels at the equilibrium swollen state measured in the amplitude sweep mode (at a frequency of $1 \mathrm{rad} / \mathrm{s}$ ), which allows us to find the linear viscoelastic regime (LVR) in which $G^{\prime}$ of the hydrogel is independent of the strain. It can be clearly observed that $G^{\prime}$ is nearly one order of magnitude higher than $G$ " for all the fabricated hydrogels, which indicates the viscoelastic nature of all hydrogels are dominated by an elastic response in the LVR. ${ }^{54}$ All the fabricated hydrogels exhibited type III (weak strain overshoot) behavior of complex fluids in which the network is made of junctions where the inter and intramolecular interactions are localized. ${ }^{55}$ The dropping in $G^{\prime}$ with the magnitude of strain amplitude indicates the rupture of networks, whereas the increase in $G$ ', indicates the ability of network reformation, which is a characteristic of type III classification, and suggests strong intermolecular interactions between RSF and nanocellulose molecules. ${ }^{55}$ Moreover, the critical strain limit (at which $G^{\prime}$ drops) of pristine RSF hydrogel is increased with nanocellulose incorporation. This supports our hypothesis that the incorporation of 
nanocellulose into the RSF hydrogel matrix decelerates the crack propagation there by increasing the strain limit (i.e. extensibility). Figure 4B shows the frequency dependency of all the fabricated hydrogels at a strain amplitude of $0.05 \%$, which is in the LVR. It can be clearly observed that G' and G', of all the hydrogels exhibit a nearly frequency independent response (indicating gel behavior) in the whole measurement range. In general, the magnitude of $G^{\prime}$ represents the stiffness of the hydrogel, whereas $G^{\prime \prime} / G^{\prime}$ or $\tan (\delta)$ represents the elastic behavior, where an ideal elastomer possesses low tan $(\delta)$ values. ${ }^{54}$ For a better understanding of the viscoelastic behavior, the G' (Figure 4C) and tan ( $\delta$ ) (Figure 4D) values of all the fabricated hydrogels measured at $1 \mathrm{rad} / \mathrm{s}$ frequency and $0.05 \%$ strain were compared at both strain and frequency modes. It is observed that the RSF/nanocellulose composites hydrogels exhibited relatively higher $\mathrm{G}^{\prime}$ values (in the range of $0.81-1.45 \mathrm{MPa}$ ) compared to pristine RSF hydrogel $(0.43 \mathrm{MPa})$. This increase in $\mathrm{G}^{\prime}$ of RSF hydrogel with incorporation of nanocellulose can be attributed to an increase in the overall sample rigidity, intermolecular interactions between RSF and nanocellulose, and network complexity by nanocellulose flocculation or entanglement. ${ }^{56}$ The $G^{\prime}$ values of equilibrium water swollen RSF/nanocellulose composites hydrogels were noticed to follow the trend RSF/BC > $\mathrm{RSF} / \mathrm{CNC}>\mathrm{RSF} / \mathrm{CNF}$, which embraces the order observed for nanocellulose crystallinity and fibrils or bundle diameter. In general, rheological and mechanical performances are primarily dictated by the capacity of the cellulose to form an organized structure. The aspect ratio or the length and diameter $(\mathrm{L} / \mathrm{d})$ ratio of the cellulose particle and its interaction with the matrix control these properties. Moreover, the RSF/nanocellulose composite hydrogels demonstrated decreased tan $(\delta)$ values compared to pristine RSF hydrogel, which indicates increased elasticity. ${ }^{54}$ The elasticity of RSF/nanocellulose composite hydrogels follows the order of $\mathrm{RSF} / \mathrm{CNF}>\mathrm{RSF} / \mathrm{BC}>\mathrm{RSF} / \mathrm{CNC}$, which is in clear alignment with the order observed for length of the nanocellulose. The fiber form of cellulose (CNF \& BC) 
demonstrated high elasticity and critical strain limit when compared to whisker form (CNC) due to the chain entanglements. ${ }^{57}$ These results are also in line with their aspect ratios. Furthermore, the obtained shear storage moduli of RSF/nanocellulose composite hydrogels were in the close range of articular cartilage tissue $(0.2-2.0 \mathrm{MPa})$ along with the similar damping characteristics. ${ }^{58}$ The higher storage modulus and lower tan $(\delta)$ values of the $\mathrm{RSF} /$ nanocellulose composite hydrogels is anticipated to increase their overall toughness compared to pristine RSF hydrogel. Therefore, the stiffness and elasticity of RSF/nanocellulose composite hydrogels can be tuned based on the type of nanocellulose used as a filler or reinforcing agent.
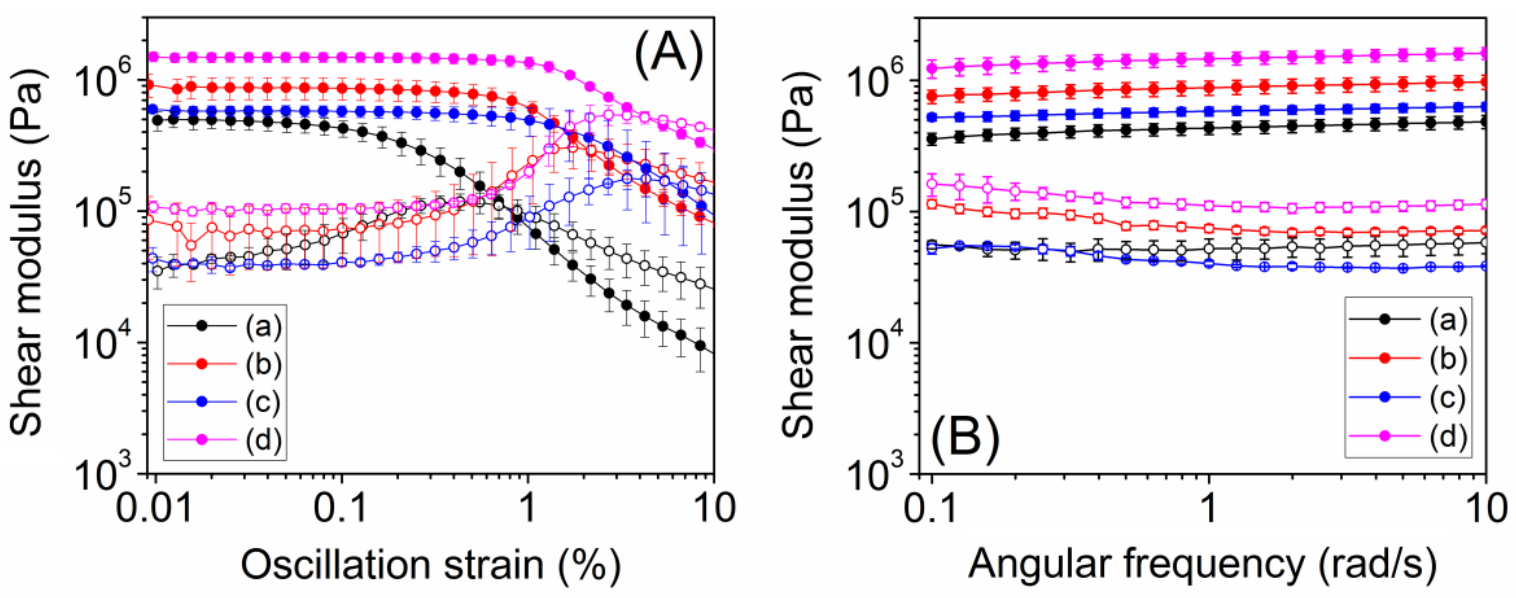

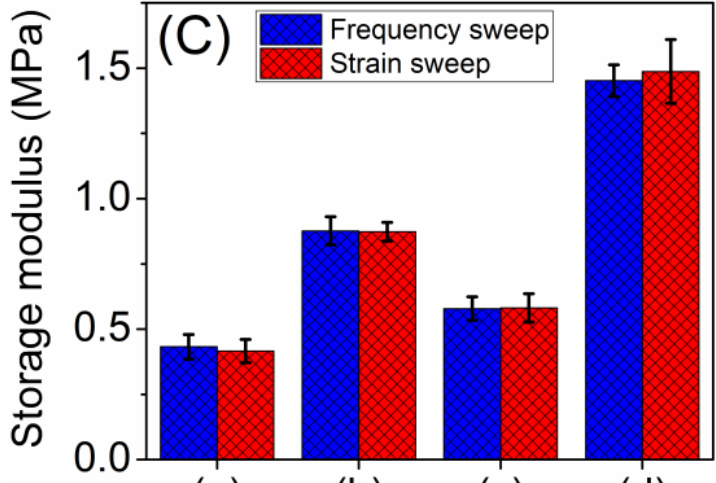

(a) (b) (c)

(d)
0.00

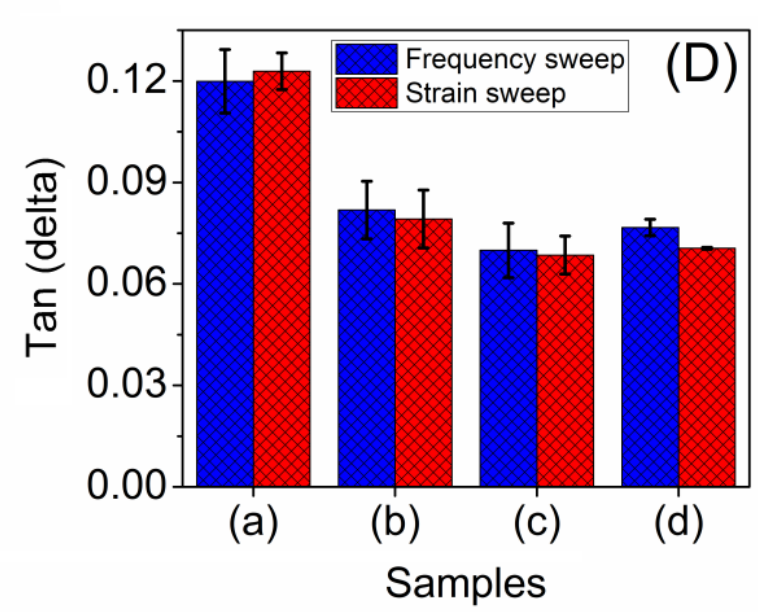

Samples

Figure 4. Storage modulus (solid symbol) and loss modulus (open symbol) of equilibrium water swollen hydrogels measured in (A) strain sweep and (B) frequency sweep mode. (C) 
and (D) are storage modulus and tan $(\delta)$, respectively measured at 0.05 strain $\%$ and $1 \mathrm{rad} / \mathrm{s}$. The sample codes are (a) RSF hydrogel, (b) RSF/CNC composite hydrogel, (c) RSF/CNF composite hydrogel and (d) RSF/BC composite hydrogel.

\section{Tensile and compressive mechanical properties of fabricated hydrogels}

The influence of different types of nanocellulose on the tensile mechanical properties of the fabricated RSF/nanocellulose composite hydrogels was investigated using tensile testing. Figure 5A shows the uniaxial tensile stress-strain curve of the equilibrium swollen composite hydrogels. The non-Hookean nature of the curve manifests complex mechanical behavior associated with their level of hydration, degree of crystallinity, inter-molecular interaction and hierarchical gel structure. ${ }^{59}$ At higher strain, the observed increase in stress could be ascribed to ordering of the polymer chain, enhanced hydrogen bonding between aligned polymer chains, physical entanglement as well as chemical bonding of elongated chains. ${ }^{8}$ The tensile mechanical properties determined from the stress vs. strain plots of the composite hydrogels were reported in Table 2. In general, Young's modulus measures the reluctance of a material to elastic deformation (i.e. higher the Young's modulus higher the stiffness), whereas ultimate tensile strength measures the resistance of a material to breaking under tension. ${ }^{59}$ The pristine RSF hydrogel exhibits a Young's modulus of $\sim 2.2 \mathrm{MPa}$ and tensile strength of $\sim 0.3 \mathrm{MPa}$, whereas the RSF/nanocellulose composites hydrogels demonstrate significantly higher modulus and strength in the range of 9.4-14.0 $\mathrm{MPa}$ and $0.9-1.1 \mathrm{MPa}$, respectively. The Young's moduli of RSF/nanocellulose composites hydrogels are seen to follow the trend $\mathrm{RSF} / \mathrm{BC}>\mathrm{RSF} / \mathrm{CNC}>\mathrm{RSF} / \mathrm{CNF}$, which is consistent with the order observed for nanocellulose crystallinity and hydrogel shear storage modulus. Moreover, the obtained Young's moduli of RSF/nanocellulose composites hydrogels were comparable to the modulus of native femoral artery $(\sim 9 \mathrm{MPa}),{ }^{60}$ and greater than that of $\mathrm{RSF} /$ graphene oxide composite hydrogel $(\sim 8.0 \mathrm{MPa}){ }^{8}$ The increase in RSF hydrogel tensile strength with 
nanocellulose incorporation may be due to an increase in intermolecular interactions between RSF and nanocellulose through strong hydrogen bonding. ${ }^{8,24}$ The tensile strength of $\mathrm{RSF} /$ nanocellulose composites hydrogels follows the order $\mathrm{RSF} / \mathrm{BC}>\mathrm{RSF} / \mathrm{CNF}>$ $\mathrm{RSF} / \mathrm{CNC}$, which is in general agreement with the order observed for the nanocellulose crystallite size and the highly ordered total $\beta$-sheet crystal structures formed in the composites. The tensile strength of the composites reinforced with the fiber form of cellulose (BC \& $\mathrm{CNF})$ is more when compared to whisker form $(\mathrm{CNC})$ due to the high aspect ratio of $\mathrm{BC}$ and $\mathrm{CNF}$ which can favor the fiber entanglements with the silk fibroin network. ${ }^{57}$ Moreover, the ultimate strain (elongation) of pristine RSF hydrogel was measured to increase with nanocellulose incorporation, which may be due to the reorientation of nanocellulose during strain enabling it to act as a decelerator that prevents the immediate fragmentation of the hydrogel. The elongation at break of RSF/nanocellulose composite hydrogels follows the order $\mathrm{RSF} / \mathrm{CNF}>\mathrm{RSF} / \mathrm{BC}>\mathrm{RSF} / \mathrm{CNC}$ which is in complete agreement with the elasticity ( $\tan \delta$ ) values obtained from rheology. The pristine RSF hydrogel exhibits a tensile toughness of $\sim 16.7 \mathrm{~kJ} / \mathrm{m}^{3}$, which is consistent with the previous report. ${ }^{7}$ The tensile toughness of pristine RSF hydrogel increased with the nanocellulose incorporation, and RSF/nanocellulose composite hydrogels exhibited tensile toughness in the range of $84.7-108.3 \mathrm{~kJ} / \mathrm{m}^{3}$. The observed trend in tensile toughness of RSF/nanocellulose composites hydrogels follows the order $\mathrm{RSF} / \mathrm{BC}>\mathrm{RSF} / \mathrm{CNF}>\mathrm{RSF} / \mathrm{CNC}$, which is in alliance with the order observed for hydrogel tensile strength. The obtained toughness of RSF/nanocellulose composite hydrogels are significantly higher than the double network hydrogels constructed from Agar, Polyacrylamide (PAAm) and Alginate $\left(3.86 \mathrm{~kJ} / \mathrm{m}^{3}\right)$, and dual crosslinked polyacrylamide/CNF hydrogel $\left(\sim 47 \mathrm{~kJ} / \mathrm{m}^{3}\right) .{ }^{61-62}$ 

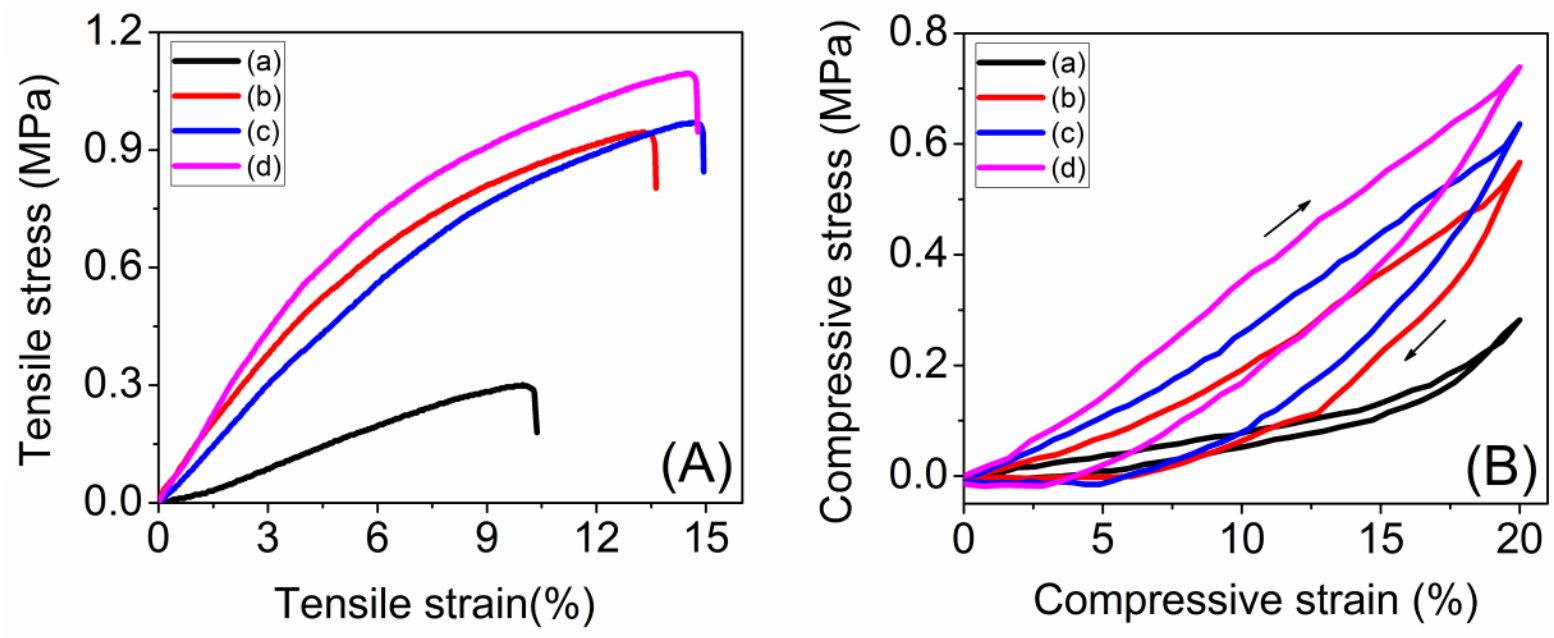

Figure 5. (A) Tensile and (B) compressive stress-strain curves of equilibrium water swollen hydrogels. The sample codes are (a) RSF hydrogel, (b) RSF/CNC composite hydrogel, (c) $\mathrm{RSF} / \mathrm{CNF}$ composite hydrogel and (d) RSF/BC composite hydrogel.

The compressive mechanical properties of the swollen hydrogels were evaluated using a small probe indentation approach rather than the plate compression, as the indentation protocol is limited by load rather than by displacement and is reported to provide more appropriate and reproducible diagnostic for mammalian tissues. ${ }^{63}$ Figure $5 \mathrm{~B}$ shows the compressive (upto $20 \%$ strain) stress-strain hysteresis loop of equilibrium swollen composite hydrogels. The compressive mechanical properties evaluated from the measured data are shown in Table 2. The pristine RSF hydrogel exhibited a compression modulus of $\sim 1.3 \mathrm{MPa}$ and strength of $\sim 0.3 \mathrm{MPa}$, whereas the $\mathrm{RSF} /$ nanocellulose composite hydrogels demonstrated significantly higher modulus and strength in the range of 2.7-3.6 $\mathrm{MPa}$ and 0.5-0.7 $\mathrm{MPa}$, respectively. The compressive modulus and strength of RSF/nanocellulose composites hydrogels were noticed to follow the order RSF/BC $>\mathrm{RSF} / \mathrm{CNF}>\mathrm{RSF} / \mathrm{CNC}$, which is in acquiescence with the order observed for nanocellulose crystallite size and the total ordered $\beta$-sheet content calculated from FTIR. The compression moduli of RSF/nanocellulose composite hydrogels were comparable to human medial meniscus tissue (0.32-1.04 MPa), ${ }^{64}$ and significantly higher than the human lumbar spinal disc annulus fibers $(0.075-0.11$ 
$\mathrm{MPa}) .{ }^{65}$ The pristine RSF hydrogel exhibited a dissipation energy of $\sim 3.9 \mathrm{~kJ} / \mathrm{m}^{3}$, whereas the RSF/nanocellulose composite hydrogels demonstrated significantly higher energy dissipation in the range of $18.7-23.6 \mathrm{~kJ} / \mathrm{m}^{3}$, which supports our hypothesis that incorporation of nanocellulose toughens the RSF hydrogel matrix via mechanical energy dissipation mechanism. ${ }^{66}$ The dissipated energy of RSF/nanocellulose composites hydrogels follows the order $\mathrm{RSF} / \mathrm{BC}>\mathrm{RSF} / \mathrm{CNF}>\mathrm{RSF} / \mathrm{CNC}$, which is in complete alignment with the order observed for hydrogel tensile strength and toughness.

Table 2. Tensile and compressive mechanical properties of fabricated hydrogels.

\begin{tabular}{|c|c|c|c|c|c|}
\hline \multirow[t]{2}{*}{ Test } & \multirow[t]{2}{*}{ Measured properties } & \multicolumn{4}{|c|}{ Fabricated hydrogels } \\
\hline & & RSF & $\mathrm{RSF} / \mathrm{CNC}$ & $\mathrm{RSF} / \mathrm{CNF}$ & RSF/BC \\
\hline \multirow[t]{4}{*}{ Tensile } & Young's Modulus (MPa) & $2.16 \pm 0.10$ & $11.79 \pm 0.09$ & $9.45 \pm 0.01$ & $14.03 \pm 0.12$ \\
\hline & Ultimate Stress (MPa) & 0.29 & 0.94 & 0.97 & 1.09 \\
\hline & Ultimate strain $(\%)$ & 10.15 & 13.41 & 14.78 & 14.09 \\
\hline & Toughness $\left(\mathrm{kJ} / \mathrm{m}^{3}\right)$ & 16.65 & 84.73 & 89.90 & 108.30 \\
\hline \multirow[t]{3}{*}{$\begin{array}{l}\text { Micro- } \\
\text { indentation }\end{array}$} & $\begin{array}{l}\text { Compressive Modulus } \\
(\mathrm{MPa})\end{array}$ & $1.29 \pm 0.05$ & $2.73 \pm 0.01$ & $3.08 \pm 0.01$ & $3.59 \pm 0.03$ \\
\hline & $\begin{array}{l}\text { Compressive strength at } \\
20 \% \text { strain }(\mathrm{MPa})\end{array}$ & 0.28 & 0.57 & 0.64 & 0.74 \\
\hline & $\begin{array}{l}\text { Energy dissipated for } 20 \% \\
\text { strain }\left(\mathrm{kJ} / \mathrm{m}^{3}\right)\end{array}$ & 3.95 & 18.70 & 22.50 & 23.58 \\
\hline
\end{tabular}

\section{Attachment, viability and proliferation of fibroblast cells on fabricated \\ hydrogels}

Cellular response is critical for a biocompatibility evaluation of biomaterials for tissue engineering applications. ${ }^{67}$ Therefore, to evaluate the biocompatibility of fabricated biomimetic hydrogels, various cellular responses, such as attachment, viability and 
proliferation were investigated using L929 fibroblast cell line. It has been reported that CNF show more pronounced cytotoxicity and oxidative stress responses in human lung epithelial A549 cells than $\mathrm{CNC}$, whereas $\mathrm{CNC}$ has caused more inflammatory response compared to $\mathrm{CNF}^{68}$ On the other hand, BC neither induced any apoptosis and necrosis nor stimulate immune response in human umbilical vein endothelial cells. ${ }^{69}$ Therefore, to assess the influence of different types of nanocellulose incorporation into RSF hydrogel matrix on biological activity, the L929 fibroblast cell line was seeded on the surface of the fabricated hydrogels. The MTS assay was employed to quantitatively investigate the cell metabolic activity of L929 cells on Tissue culture plate (TCP) and fabricated hydrogels, whereas the Trypan Blue staining was used to access the cell viability after culturing for 1,3 and 5 days. Figure 6A shows the measured absorbance signal from the extracted media of fabricated hydrogels and TCP control on days 1, 3 and 5 after incubating with MTS reagent. It is observed that all the hydrogels exhibited a strong absorbance signal on each day indicating the suitability of the hydrogel surface for cell proliferation, where the absorption coefficient is proportional to the cell proliferation. The increase in absorbance signal with number of days indicates the cells are viable and capable of proliferation. However, the cell proliferation (absorbance signal) on $\mathrm{RSF} / \mathrm{CNC}$ composite hydrogel on each day was relatively lower among the fabricated hydrogels, which may be due to its low swelling ability and decreased pore size, which might hinder the free flow of cells and nutrients. ${ }^{70}$ Similar decrease in proliferation is demonstrated by L929 cells on less swollen chitosan/alginate gels when compared with the highly swollen gel. ${ }^{71}$ Moreover, the cell viability evaluated using trypan blue assay demonstrated excellent viability close to $90 \%$, which indicates that all the hydrogels are highly biocompatible (Figure 6B). No noticeable dissolution or degradation of the RSF/NF hydrogel was observed after 5 days of cell culture. 


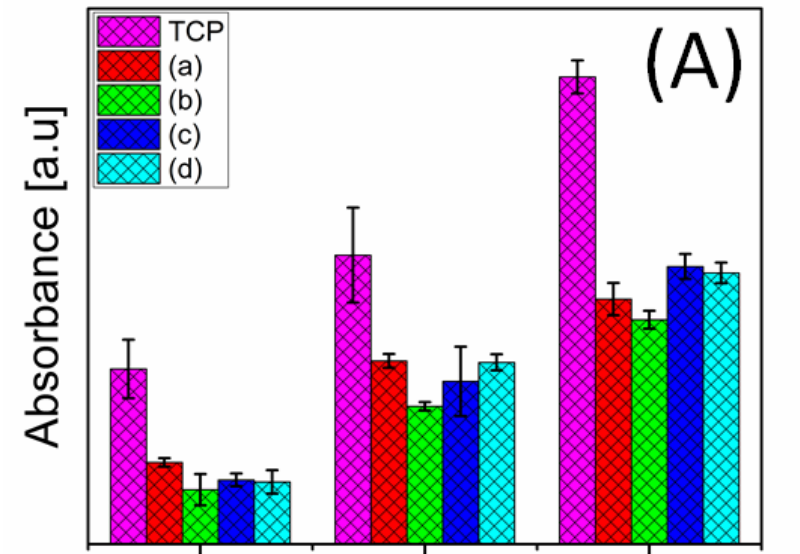

Day1

Day3

Day5

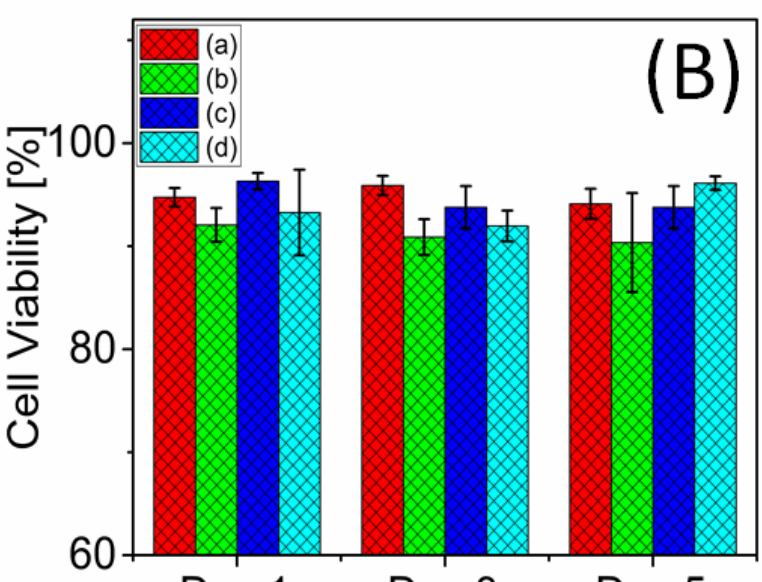

Day 1

Day 3

Day 5

Figure 6. (A) Absorbance signal from MTS assay (B) Quantitative estimation of the cell variability using Trypan Blue. The sample codes are (TCP) Tissue culture plate, (a) RSF hydrogel, (b) RSF/CNC composite hydrogel, (c) RSF/CNF composite hydrogel and (d) RSF/BC composite hydrogel.

SEM images in Figure 7 show the cell attachment behavior with time on the fabricated hydrogel surfaces. The images of cell adhered gels do not show high degree of porosity due to the glutaraldehyde-based chemical cell fixing method. This is in line with previous observation where chemical fixation followed by systematic dehydration with ethanol has been reported to influence the morphological feature of gel. ${ }^{72}$ The number of cells attached on the hydrogel surfaces is seen to increase with time; with day 1 study showing the circular shape, whereas day 3 studies demonstrate cell attachment on gel surface with spindle shape. The cells cultured for 5 days show excellent attachment and spreading on all the hydrogel surfaces. The difference in the number of cells attached on day 3 and day 5 is likely due to the detachment of weakly attached cells during several washing steps during cell fixation. The number of cells attached on the surface of RSF/CNF and RSF/BC composite hydrogels on day 5 is higher when compared to pristine RSF and RSF/CNC composite hydrogels, which may be due to the characteristics of L929 cells, which preferentially grows on surfaces with a higher modulus. ${ }^{73}$ Overall, it is evident that the incorporation of 
nanocellulose does not induce appreciable cytotoxicity on RSF hydrogel and demonstrate the cell viability and ability to support cellular activities such as attachment and proliferation over a period of time. Among the fabricated hydrogels, the RSF/CNF and RSF/BC composite hydrogels demonstrate the most suitable matrix for encouraging fibroblast cell proliferation with good balance between water uptake, pore size and structure, and modulus.
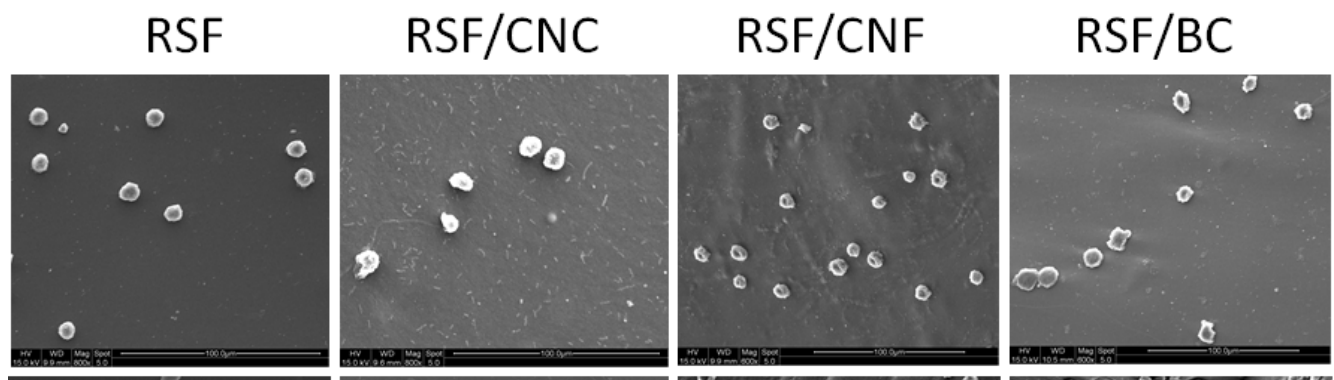

\section{Day 1}
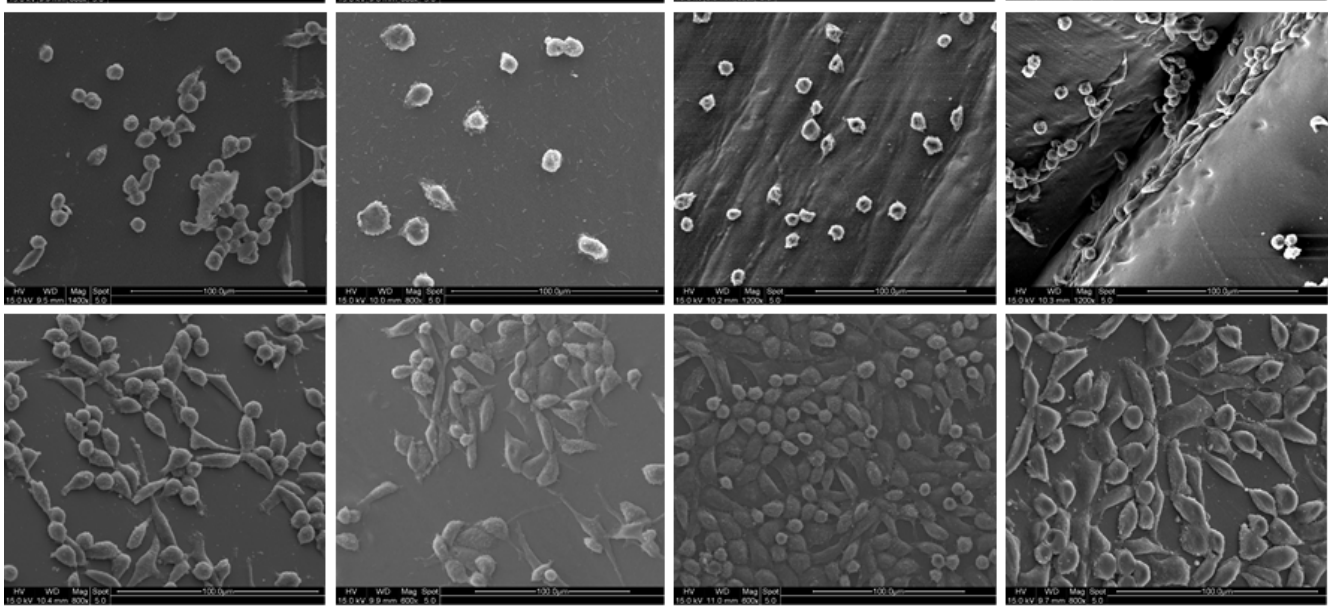

Day 3
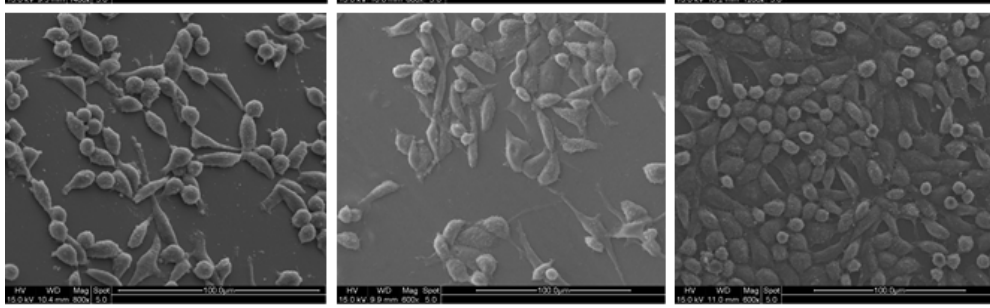

Day 5

Figure 7. SEM images illustrating the cell attachment progress with respect to time (days).

\section{Preliminary printing ability of RSF/nanocellulose inks}

The photocrosslinked RSF/nanocellulose composite hydrogels demonstrated excellent biocompatibility and tunable structural and mechanical properties demonstrating their potential for a wide range of tissue engineering applications. However, each application needs a specific scaffold design which is highly difficult to achieve by conventional fabrication approach. ${ }^{19}$ 3D printing is an advanced fabrication technique that is revolutionizing the current medical research by making the customized designs using 
hydrogels to match the target tissue architecture. ${ }^{74}$ However, like other fabrication methods, 3D printing methods are also limited by the resolution of the device, setting a minimum length scale. To print a customized architecture, understanding and testing the preliminary printability of the precursor material is critical. Therefore, to evaluate the printability, the flow properties of hydrogel forming solutions (inks) of RSF/nanocellulose composites and pristine RSF were investigated using rotational rheology. Rheology is an important tool, which can act as an interface to bridge the gap between conventional fabrication and 3D printing. Figure $8 \mathrm{~A}$ shows the apparent viscosity profile of RSF and RSF/nanocellulose composite inks with respect to shear. All the samples show a decrease in viscosity with an increase in shear rate (shear thinning) between the experimental shear rate $\left(0.02\right.$ and $\left.10 \mathrm{~s}^{-1}\right)$, which is a critical parameter for extrusion-based 3D printing; where the viscous ink deforms slowly under shear providing good printing resolution. ${ }^{75}$ For shear-thinning fluids, the influence of shear rate on viscosity can be described using the following power-law model. ${ }^{76}$

$$
\eta=K \gamma^{n-1}
$$

where $\eta$ is the viscosity, $\mathrm{K}$ is the flow consistency index, $\gamma$ is the shear rate, and $\mathrm{n}$ is the flow behavior index. The $\mathrm{K}$ and $\mathrm{n}$ values calculated by fitting the curves to equation 8 (Figure S4 in Supporting Information) are reported in Table S1 of the Supporting Information. The flow index behavior of all samples was estimated to be $n<1$, which is a characteristic of shearthinning behavior. The higher $\mathrm{K}$ values of RSF/nanocellulose composite ink systems indicate the improvement in ink consistency. Moreover, the minimally inflammatory and non-toxic photocrosslinking method employed in the current study provides the opportunity for using the RSF/nanocellulose composite ink systems as bioinks for 3D printing with cells. ${ }^{75,77}$ Blending nanocellulose with RSF increased the viscosity of the composites in the order of $\mathrm{RSF} / \mathrm{BC}>\mathrm{RSF} / \mathrm{CNF}>\mathrm{RSF} / \mathrm{CNC}$. The increase in viscosity may be attributed to several 
parameters including intermolecular interactions between RSF and nanocellulose, and nanocellulose dimension, crystallinity, orientation and entanglement. ${ }^{24,78}$ Moreover, $\mathrm{RSF} /$ nanocellulose composite inks exhibit viscosity in the range of $0.67 \times 10^{3}-6.90 \times 10^{6} \mathrm{~Pa} . \mathrm{s}$ at low shear rates of $0.05-0.10 \mathrm{~s}^{-1}$, which is a suitable range for the printing of biomaterials through extrusion. ${ }^{79}$ In extrusion printing, the pressure applied will induce shear on the ink, which is maximum at the walls of the nozzle $\left(\gamma_{\max }\right)$ and can be estimated from the following equation: ${ }^{80}$

$$
\gamma_{\max }=\frac{4 S}{\pi r}
$$

where $\mathrm{S}$ is the printing speed and $\mathrm{r}$ represents the radius of the nozzle. Using equation 9, it was estimated (see experimental section for optimized printing parameters) that the maximum shear rate experienced by the ink was $\sim 87 \mathrm{~s}^{-1}$. It is important that after experiencing such high shear rate the ink should regain its original strength (structure) once it is extruded through the nozzle. Therefore, the structural recovery behavior of inks (Figure 8B) was studied by varying the shear rates (low-high-low: $0.1 \mathrm{~s}^{-1}-90 \mathrm{~s}^{-1}-0.1 \mathrm{~s}^{-1}$ ) with respect to time. All the inks demonstrated acceptable recovering ability (within few seconds), and the recovery percentage was observed in the order of $\mathrm{RSF} / \mathrm{CNC}(\sim 90.5 \%)>\mathrm{RSF} / \mathrm{BC}$ $(\sim 74.2 \%)>\operatorname{RSF}(\sim 53.6 \%)>\mathrm{RSF} / \mathrm{CNF}(\sim 53.1 \%)$. The rapid viscosity recovery behavior may be influenced or affected by several parameters including intermolecular interactions between RSF and nanocellulose, and nanocellulose dimension, crystallinity, orientation and entanglement. ${ }^{24,78}$ Therefore, the observed shear-thinning nature and structural recovery of the composite inks strongly support their potential in $3 \mathrm{D}$ printing. ${ }^{75}$ In addition, photocurable 3D printing of polymeric solutions offers economical and production benefits, where the in situ crosslinking of extruded solution can be performed simultaneously during the printing process. ${ }^{81}$ Therefore, we employed photocuring rheology to investigate the in situ 
photocrosslinking reaction potential and kinetics of our RSF/nanocellulose composite hydrogel inks. The storage modulus of all samples increased drastically within 30 seconds of exposure to light (Figure S5 in Supporting Information), indicating the potential of simultaneous crosslinking of tested systems to form gels during 3D printing. The observed difference in storage modulus of crosslinked gels compared to equilibrium water swollen hydrogels can be attributed to their water uptake capacity and applied compression $(10 \%)$. Similar, photocrosslinking kinetics has also been reported for 3D printing and crosslinking of Gelatin Methacryloyl (GelMA) bio-inks using Irgacure. ${ }^{82}$
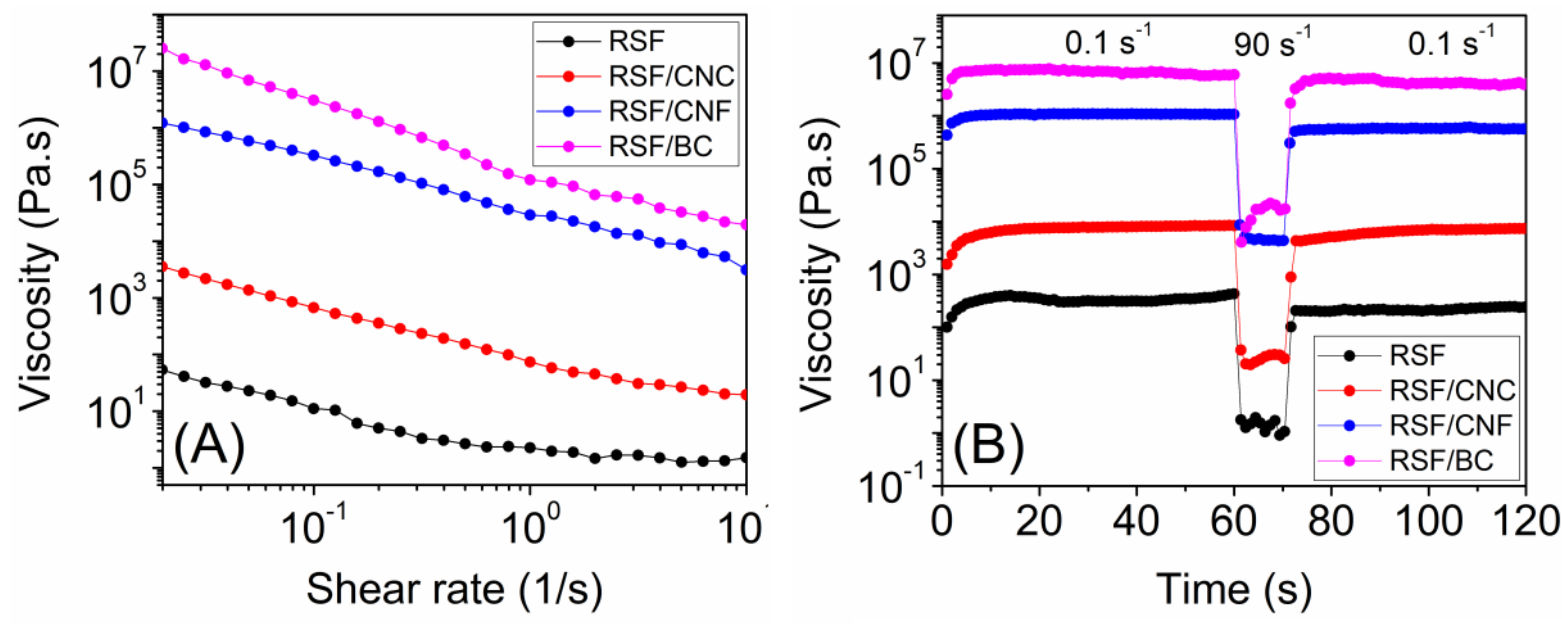

Figure 8. (A) Shear rate dependent viscosity profile and (B) Structural recovery behavior of hydrogel forming RSF and RSF/nanocellulose composite solutions (inks).

To investigate the 3D printability of hydrogel forming $\mathrm{RSF} /$ nanocellulose composite inks, several printing variables were tested and the optimized parameters (see experimental section) were used to demonstrate printing. Figure 9A shows the 3D printed single and double layers of RSF and RSF/nanocellulose composite hydrogels. The double layers were printed by curing the first layer using white light for 40 seconds before printing the second layer and curing to ensure complete crosslinking. For good shape fidelity of 3D printed hydrogels, the diameter (width) of the 3D printed strand/filament should be equal to the diameter of the printing nozzle. However, the tested viscoelastic inks tended to spread (relax) 
once they were extruded from the nozzle. Therefore, the strand width variation of the printed gel from the nozzle size (Figure 9A) is attributed to material relaxation after extrusion. Among the printed constructs, it was observed that the strand width is marginally lower for $\mathrm{RSF} / \mathrm{CNC}$ and $\mathrm{RSF} / \mathrm{BC}$ composite inks compared to RSF ink. This decrease in strand width can be ascribed to the increase in structural recovery of the respective composite inks revealed by the rheological measurements. A similar decrease in strand width has been reported for alginate/graphene oxide 3D printed construct with the increase in graphene oxide concentration. ${ }^{23}$ The authors aimed to assess and improve the 3D printability of hydrogel forming composite inks by optimizing several parameters. At higher concentration, printability is seen to improve, however higher viscosity affects feature resolution. Thus, printing feature with higher resolution (in micron range) becomes difficult at high concentration. The 3D printed strand width of 1-1.5 mm (Figure S6) is in the range reported for silk fibroin-based 3D printed gels. ${ }^{20}$ In order to quantify the influence of different types of nanocellulose on 3D printing fidelity of RSF/nanocellulose composite hydrogel systems, the printing accuracy of the composite inks were calculated (Figure 9B). It is observed that the printing accuracy of the RSF constructs marginally improved with the incorporation of CNC and $\mathrm{BC}$ and decreased with CNF. Several parameters including intermolecular interactions between RSF and nanocellulose, and the nanocellulose dimension, crystallinity, orientation and entanglement influence the accuracy. ${ }^{24,78}$ The printing accuracy of RSF/nanocellulose composite hydrogels followed the trend $\mathrm{RSF} / \mathrm{CNC}>\mathrm{RSF} / \mathrm{BC}>\mathrm{RSF} / \mathrm{CNF}$, which is in complete agreement with the order observed for viscosity (structure) recovery. In addition, the rod form of $\mathrm{CNC}$ has a tendency to reorganize themselves from random to oriented structures in alignment with the flow direction when subjected to shear which is an important factor in improving the printability. ${ }^{83}$ Therefore, the incorporation of nanocellulose not only improved the structural, mechanical and biological properties of RSF hydrogel but also its 3D 
printability. Efforts were also made to 3D print hydrogels continuously by shining light simultaneously. However, clogging of the solution on the nozzle was observed after printing 3 layers, suggesting further optimization was required of the concentration of crosslinking agent, protein concentration and flow rate to match the crosslinking kinetics. A detailed investigation on 3D printing of complex composite scaffold structures, and their structural mechanical, degradation and biological property investigation are beyond the scope of this article and will be reported in future.

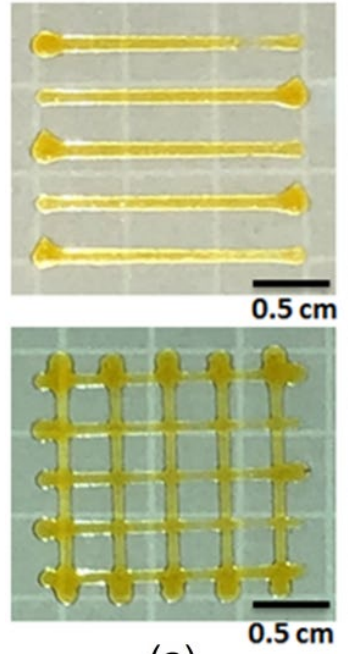

(a)
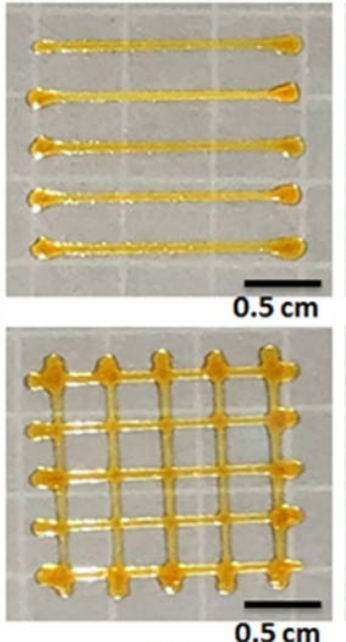

(b)
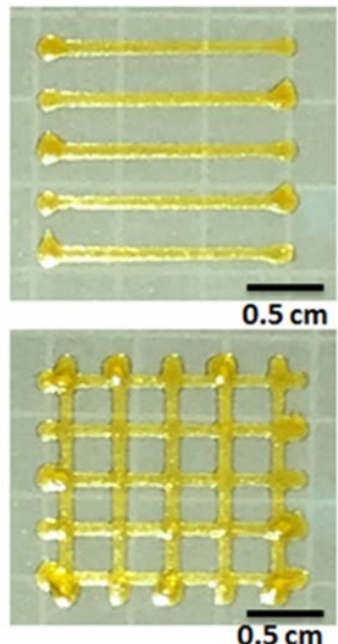

(c)

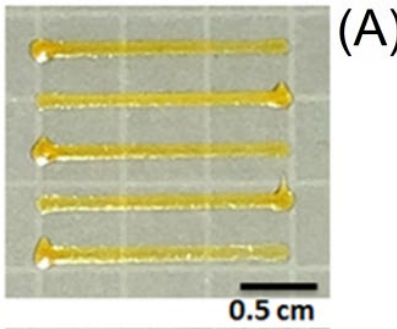

(A)

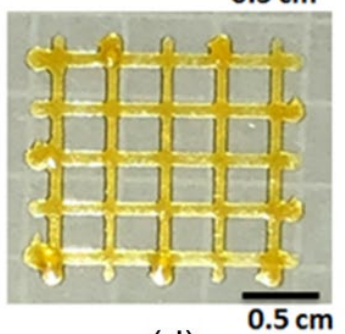

(d)

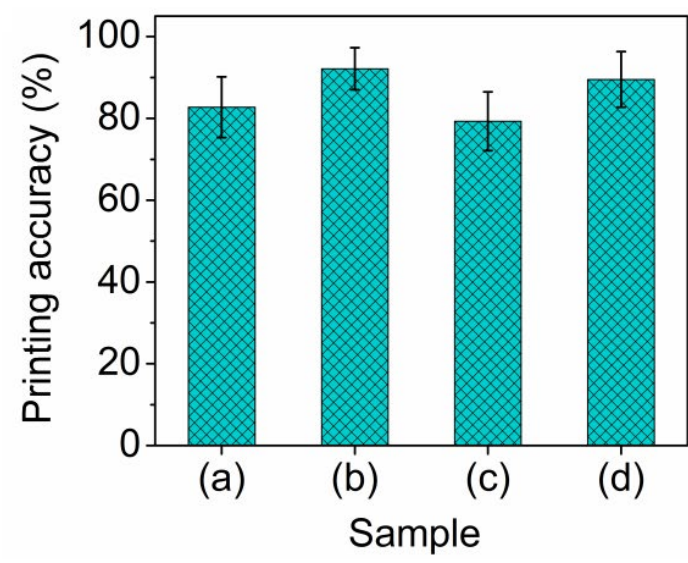

(B)

Figure 9. Optical images of single and double layered 3D printed fabricated hydrogels (A), and their calculated printing accuracy (B). The sample codes are (a) RSF hydrogel, (b) RSF/CNC composite hydrogel, (c) RSF/CNF composite hydrogel and (d) RSF/BC composite hydrogel. 
In a macroscopic sample, the molecular building blocks transmit the properties across different length scales. In the fabricated RSF/nanocellulose composite systems, the physicochemical properties of hydrogels/inks are largely influenced by the physical property of the nanocellulose used. The overall order of properties of the fabricated biomimetic hydrogels is presented in Table S2 in Supporting Information. Given the complexity of the fabricated composite hydrogel system, it is extremely difficult to exactly quantify the contributions of each type of nanocellulose to the overall hydrogel property; especially biocompatibility, where cell adhesion and proliferation depends on several factors, such as pore size/structure/alignment, hydrophobicity, stiffness, etc. However, from the fundamental perspective, conclusions can be drawn based on the physical characteristics of different types of nanocellulose used, which largely influence the physical property changes and performance of fabricated biomimetic hydrogels. The inter-hydrophobic domain distance and the resulting water uptake property of hydrogels were observed to be largely influenced by the surface area of nanocellulose used, where larger the surface area of nanocellulose, more the RSF-cellulose interaction. The shear storage modulus and Young's modulus measured at low mechanical strain were observed to be largely influenced by the crystallinity index of nanocellulose used. Conversely, the overall tensile toughness, compression modulus and

energy dissipated were observed to be largely influenced by the crystallite size of nanocellulose used, where higher $\beta$-content was also measured in direct relation to larger crystallite size. Furthermore, the printability of composite inks were observed to be largely influenced by inter-nanocellulose interaction, where higher the aspect ratio and flexibility, higher the possibility of chain entanglement.

\section{CONCLUSIONS}

In summary, RSF/nanocellulose biomimetic hydrogels were successfully fabricated using photocrosslinking method. The effects of different types of nanocellulose, such as CNC, CNF 
and $\mathrm{BC}$ on different aspects like structural (micro, secondary and hierarchical), mechanical, biological and 3D printing ability is investigated for the first time. The incorporation of nanocellulose into chemically crosslinked RSF matrix significantly influenced the water uptake capacity, micropore size and structure, inter-hydrophobic domain distance, $\beta$-sheet content, viscoelasticity, tensile and compressive mechanical properties, biocompatibility and 3D printability. The fabricated hydrogels demonstrated superior mechanical performance when compared with other polymer hydrogels and natural tissue constructs in terms of shear, tensile and compression. Fibroblast cell line was used in this study to perform preliminary screening of biocompatibility and cell attachment potential of fabricated hydrogel systems to be applied in a wide range of tissue engineering applications. However, appropriate biological studies using a desired cell line needs to be investigated for the suitability of these hydrogels for other specific application. Based on the experimental evidence, it is conclusive that each type of nanocellulose has its own significance in improving the desired property and resulting performance of the fabricated $\mathrm{RSF} /$ nanocellulose composite hydrogels. To summarize, the RSF/nanocellulose composite hydrogel containing BC demonstrated superior mechanical performance, whereas the one with CNF demonstrated relatively excellent biological performance, and the one with $\mathrm{CNC}$ demonstrated improved printability. Therefore, in addition to better understanding of the tunability of structure and performance of these biomimetic gels, new insights into the initial printability of these gels has potential for a broad range of soft and hard tissue engineering applications.

\section{Author Contributions}

The manuscript was written through contributions of all authors. All authors have given approval to the final version of the manuscript.

\section{Notes}


The authors declare no competing financial interest.

\section{ACKNOWLEDGEMENTS}

This research has been financially supported by the Australian Research Council (ARC) through Discovery research grant (DP160101267). Access to the SANS and USANS facility at the Australian Centre for Neutron Scattering was supported through ANSTO beam time award (P5772). The authors acknowledge the facilities, scientific and technical assistance of the Adelaide Microscopy (The University of Adelaide, Adelaide), BioFab3D (St Vincent's Hospital, Melbourne), Micro-Nano Research Facility, Centre for Nanoscale Biophotonics and Microscopy and Microanalysis Facility (RMIT University, Melbourne). This work benefited from the use of SasView application, originally developed under NSF award DMR-0520547. SasView contains code developed with funding from the European Union's Horizon 2020 research and innovation programme under the SINE2020 project, grant agreement No 654000.

\section{REFERENCES}

(1) Jonker, A. M.; Löwik, D. W. P. M.; van Hest, J. C. M., Peptide- and Protein-Based Hydrogels. Chem. Mater. 2012, 24, 759-773, DOI: 10.1021/cm202640w.

(2) Radhakrishnan, J.; Subramanian, A.; Krishnan, U. M.; Sethuraman, S., Injectable and 3D Bioprinted Polysaccharide Hydrogels: From Cartilage to Osteochondral Tissue Engineering. Biomacromolecules 2017, 18 1-26, DOI: 10.1021/acs.biomac.6b01619.

(3) Kapoor, S.; Kundu, S. C., Silk protein-based hydrogels: Promising advanced materials for biomedical applications. Acta Biomater. 2016, 31, 17-32, DOI: 10.1016/j.actbio.2015.11.034.

(4) Chang, C.; Zhang, L., Cellulose-based hydrogels: Present status and application prospects. Carbohydr. Polym. 2011, 84, 40-53, DOI: 10.1016/j.carbpol.2010.12.023.

(5) Whittaker, J. L.; Choudhury, N. R.; Dutta, N. K.; Zannettino, A., Facile and rapid ruthenium mediated photo-crosslinking of Bombyx mori silk fibroin. J. Mater. Chem. B 2014, 2, 6259-6270, DOI: 10.1039/C4TB00698D. 
(6) Whittaker, J. L.; Dutta, N. K.; Elvin, C. M.; Choudhury, N. R., Fabrication of highly elastic resilin/silk fibroin based hydrogel by rapid photo-crosslinking reaction. J. Mater. Chem. B 2015, 3, 6576-6579, DOI: 10.1039/C5TB00970G.

(7) Whittaker, J. L.; Dutta, N. K.; Zannettino, A.; Choudhury, N. R., Engineering DN hydrogels from regenerated silk fibroin and poly(N-vinylcaprolactam). J. Mater. Chem. B 2016, 4, 5519-5533, DOI: 10.1039/C6TB01055E.

(8) Balu, R.; Reeder, S.; Knott, R.; Mata, J.; de Campo, L.; Dutta, N. K.; Choudhury, N. R., Tough Photocrosslinked Silk Fibroin/Graphene Oxide Nanocomposite Hydrogels. Langmuir 2018, 34, 9238-9251, DOI: 10.1021/acs.langmuir.8b01141.

(9) Whittaker, J. L.; Balu, R.; Knott, R.; de Campo, L.; Mata, J. P.; Rehm, C.; Hill, A. J.; Dutta, N. K.; Roy Choudhury, N., Structural evolution of photocrosslinked silk fibroin and silk fibroin-based hybrid hydrogels: A small angle and ultra-small angle scattering investigation. Int. J. Biol. Macromol. 2018, 114, 998-1007, DOI: 10.1016/j.ijbiomac.2018.03.044.

(10) Zhu, Z.; Kikuchi, Y.; Kojima, K.; Tamura, T.; Kuwabara, N.; Nakamura, T.; Asakura, T., Mechanical Properties of Regenerated Bombyx mori Silk Fibers and Recombinant Silk Fibers Produced by Transgenic Silkworms. J. Biomater. Sci., Polym. Ed. 2010, 21, 395-411, DOI: $10.1163 / 156856209 X 423126$.

(11) Courtenay, J. C.; Sharma, R. I.; Scott, J. L., Recent Advances in Modified Cellulose for Tissue Culture Applications. Molecules 2018, 23, 654, DOI: 10.3390/molecules23030654.

(12) Oliveira Barud, H. G.; Barud, H. d. S.; Cavicchioli, M.; do Amaral, T. S.; Junior, O. B. d. O.; Santos, D. M.; Petersen, A. L. d. O. A.; Celes, F.; Borges, V. M.; de Oliveira, C. I.; de Oliveira, P. F.; Furtado, R. A.; Tavares, D. C.; Ribeiro, S. J. L., Preparation and characterization of a bacterial cellulose/silk fibroin sponge scaffold for tissue regeneration. Carbohydr. Polym. 2015, 128, 41-51, DOI: 10.1016/j.carbpol.2015.04.007.

(13) Zhao, X., Multi-scale multi-mechanism design of tough hydrogels: building dissipation into stretchy networks. Soft Matter 2014, 10, 672-687, DOI: 10.1039/C3SM52272E.

(14) De France, K. J.; Hoare, T.; Cranston, E. D., Review of Hydrogels and Aerogels Containing Nanocellulose. Chem. Mater. 2017, 29, 4609-4631, DOI: 10.1021/acs.chemmater.7b00531.

(15) Nascimento, D. M.; Nunes, Y. L.; Figueirêdo, M. C. B.; de Azeredo, H. M. C.; Aouada, F. A.; Feitosa, J. P. A.; Rosa, M. F.; Dufresne, A., Nanocellulose nanocomposite 
hydrogels: technological and environmental issues. Green Chem. 2018, 20, 2428-2448, DOI: 10.1039/C8GC00205C.

(16) Huang, J.; Liu, L.; Yao, J., Electrospinning of Bombyx mori silk fibroin nanofiber mats reinforced by cellulose nanowhiskers. Fibers Polym. 2011, 12, 1002-1006, DOI: 10.1007/s12221-011-1002-7.

(17) Feng, Y.; Li, X.; Li, M.; Ye, D.; Zhang, Q.; You, R.; Xu, W., Facile Preparation of Biocompatible Silk Fibroin/Cellulose Nanocomposite Films with High Mechanical Performance. ACS Sustainable Chem. Eng. 2017, 5, 6227-6236, DOI: 10.1021/acssuschemeng.7b01161.

(18) Chen, J.; Zhuang, A.; Shao, H.; Hu, X.; Zhang, Y., Robust silk fibroin/bacterial cellulose nanoribbon composite scaffolds with radial lamellae and intercalation structure for bone regeneration. J. Mater. Chem. B 2017, 5, 3640-3650, DOI: 10.1039/C7TB00485K.

(19) Yan, Q.; Dong, H.; Su, J.; Han, J.; Song, B.; Wei, Q.; Shi, Y., A Review of 3D Printing Technology for Medical Applications. Engineering 2018, 4, 729-742, DOI: 10.1016/j.eng.2018.07.021.

(20) Wang, Q.; Han, G.; Yan, S.; Zhang, Q., 3D Printing of Silk Fibroin for Biomedical Applications. Materials 2019, 12, 504, DOI: 10.3390/ma12030504.

(21) Athukoralalage, S. S.; Balu, R.; Dutta, N. K.; Roy Choudhury, N., 3D Bioprinted Nanocellulose-Based Hydrogels for Tissue Engineering Applications: A Brief Review. Polymers 2019, 11, 898, DOI: 10.3390/polym11050898.

(22) Huang, L.; Du, X.; Fan, S.; Yang, G.; Shao, H.; Li, D.; Cao, C.; Zhu, Y.; Zhu, M.; Zhang, Y., Bacterial cellulose nanofibers promote stress and fidelity of 3D-printed silk based hydrogel scaffold with hierarchical pores. Carbohydr. Polym. 2019, 221, 146-156, DOI: 10.1016/j.carbpol.2019.05.080.

(23) Li, H.; Liu, S.; Lin, L., Rheological study on 3D printability of alginate hydrogel and effect of graphene oxide. Int. J. Bioprint. 2016, 2, 54-66, DOI: 10.18063/IJB.2016.02.007.

(24) Tian, D.; Li, T.; Zhang, R.; Wu, Q.; Chen, T.; Sun, P.; Ramamoorthy, A., Conformations and Intermolecular Interactions in Cellulose/Silk Fibroin Blend Films: A Solid-State NMR Perspective. J. Phys. Chem. B 2017, 121, 6108-6116, DOI: 10.1021/acs.jpcb.7b02838.

(25) Basu, P.; Saha, N.; Saha, P., Inorganic calcium filled bacterial cellulose based hydrogel scaffold: novel biomaterial for bone tissue regeneration. Int. J. Polym. Mater. Polym. Biomater. 2019, 68, 134-144. DOI: 10.1080/00914037.2018.1525733. 
(26) Segal, L.; Creely, J. J.; Martin, A. E.; Conrad, C. M., An Empirical Method for Estimating the Degree of Crystallinity of Native Cellulose Using the X-Ray Diffractometer. Text. Res. J. 1959, 29, 786-794, DOI: 10.1177/004051755902901003.

(27) Patterson, A. L., The Scherrer Formula for X-Ray Particle Size Determination. Phys. Rev. 1939, 56, 978-982, DOI: 10.1103/PhysRev.56.978.

(28) Truong, M. Y.; Dutta, N. K.; Choudhury, N. R.; Kim, M.; Elvin, C. M.; Nairn, K. M.; Hill, A. J., The effect of hydration on molecular chain mobility and the viscoelastic behavior of resilin-mimetic protein-based hydrogels. Biomaterials 2011, 32, 8462-73, DOI: 10.1016/j.biomaterials.2011.07.064.

(29) Wood, K.; Mata, J. P.; Garvey, C. J.; Wu, C.-M.; Hamilton, W. A.; Abbeywick, P.; Bartlett, D.; Bartsch, F.; Baxter, P.; Booth, N., QUOKKA, the pinhole small-angle neutron scattering instrument at the OPAL Research Reactor, Australia: design, performance, operation and scientific highlights. J. Appl. Crystallogr. 2018, 51, 294-314, DOI: $10.1107 / \mathrm{S} 1600576718002534$.

(30) Lipfert, J.; Doniach, S., Small-angle X-ray scattering from RNA, proteins, and protein complexes. Annu. Rev. Biophys. Biomol. Struct. 2007, 36, 307-327, DOI: 10.1146/annurev.biophys.36.040306.132655.

(31) Svergun, D. I.; Koch, M. H., Small-angle scattering studies of biological macromolecules in solution. Rep. Prog. Phys. 2003, 66, 1735-1782.

(32) Kline, S. R., Reduction and analysis of SANS and USANS data using IGOR Pro. $J$. Appl. Crystallogr. 2006, 39, 895-900, DOI: 10.1107/S0021889806035059.

(33) Konarev, P. V.; Volkov, V. V.; Sokolova, A. V.; Koch, M. H.; Svergun, D. I., PRIMUS: a Windows PC-based system for small-angle scattering data analysis. J. Appl. Crystallogr. 2003, 36, 1277-1282, DOI: 10.1107/S0021889803012779.

(34) Saffer, E. M.; Lackey, M. A.; Griffin, D. M.; Kishore, S.; Tew, G. N.; Bhatia, S. R., SANS study of highly resilient poly(ethylene glycol) hydrogels. Soft Matter 2014, 10, 19051916, DOI: 10.1039/C3SM52395K.

(35) Rehm, C.; de Campo, L.; Brule, A.; Darmann, F.; Bartsch, F.; Berry, A., Design and performance of the variable-wavelength Bonse-Hart ultra-small-angle neutron scattering diffractometer KOOKABURRA at ANSTO. J. Appl. Crystallogr. 2018, 51, 1-8, DOI: org/10.1107/S1600576717016879.

(36) Lam, T.; Hauser, N.; Götz, A.; Hathaway, P.; Franceschini, F.; Rayner, H.; Zhang, L., GumTree-An integrated scientific experiment environment. Phys. B 2006, 385, 1330-1332, DOI: $10.1016 /$ j.physb.2006.06.069. 
(37) Zhang, J.; Allardyce, B. J.; Rajkhowa, R.; Zhao, Y.; Dilley, R. J.; Redmond, S. L.; Wang, X.; Liu, X., 3D Printing of Silk Particle-Reinforced Chitosan Hydrogel Structures and Their Properties. ACS Biomater. Sci. Eng. 2018, 4, 3036-3046, DOI: 10.1021/acsbiomaterials.8b00804.

(38) Novo, L. P.; Bras, J.; García, A.; Belgacem, N.; Curvelo, A. A. S., Subcritical Water: A Method for Green Production of Cellulose Nanocrystals. ACS Sustainable Chem. Eng 2015, 3, 2839-2846, DOI: 10.1021/acssuschemeng.5b00762.

(39) Hunt, J. A.; Chen, R.; van Veen, T.; Bryan, N., Hydrogels for tissue engineering and regenerative medicine. J. Mater. Chem. B 2014, 2, 5319-5338, DOI: 10.1039/C4TB00775A.

(40) Ooi, S. Y.; Ahmad, I.; Amin, M. C. I. M., Cellulose nanocrystals extracted from rice husks as a reinforcing material in gelatin hydrogels for use in controlled drug delivery systems. Ind. Crops Prod. 2016, 93, 227-234, DOI: 10.1016/j.indcrop.2015.11.082.

(41) Kim, H. J.; Yang, Y. J.; Oh, H. J.; Kimura, S.; Wada, M.; Kim, U.-J., Cellulose-silk fibroin hydrogels prepared in a lithium bromide aqueous solution. Cellulose 2017, 24, 50795088, DOI: 10.1007/s10570-017-1491-7.

(42) Shibayama, M., Structure-mechanical property relationship of tough hydrogels. Soft Matter 2012, 8, 8030-8038, DOI: 10.1039/C2SM25325A.

(43) Balu, R.; Mata, J. P.; Knott, R.; Elvin, C. M.; Hill, A. J.; Choudhury, N. R.; Dutta, N. K., Effects of Crowding and Environment on the Evolution of Conformational Ensembles of the Multi-Stimuli-Responsive Intrinsically Disordered Protein, Rec1-Resilin: A Small-Angle Scattering Investigation. J. Phys. Chem. $B$ 2016, 120, 6490-503, DOI: 10.1021/acs.jpcb.6b02475.

(44) Schaefer, D. W.; Agamalian, M. M., Ultra-small-angle neutron scattering: a new tool for materials research. Curr. Opin. Solid State Mater. Sci. 2004, 8, 39-47, DOI: 10.1016/j.cossms.2004.01.012.

(45) Lindh, E. L.; Bergenstråhle-Wohlert, M.; Terenzi, C.; Salmén, L.; Furó, I., Nonexchanging hydroxyl groups on the surface of cellulose fibrils: The role of interaction with water. Carbohydr. Res. 2016, 434, 136-142, DOI: 10.1016/j.carres.2016.09.006.

(46) Feigin, L. A.; Svergun, D. I. Investigation of Polymer Substances. In Structure Analysis by Small-Angle X-Ray and Neutron Scattering; Taylor, G. W., Ed.; Springer, 2013; pp 187-218, DOI: 10.1007/978-1-4757-6624-0.

(47) Hammouda, B., A new Guinier-Porod model. J. Appl. Crystallogr. 2010, 43, 716719, DOI: 10.1107/S0021889810015773. 
(48) Debye, P.; Anderson Jr, H.; Brumberger, H., Scattering by an inhomogeneous solid. II. The correlation function and its application. J. Appl. Phys. 1957, 28, 679-683, DOI: 10.1063/1.1722830.

(49) Mildner, D.; Hall, P., Small-angle scattering from porous solids with fractal geometry. J. Phys. D: Appl. Phys. 1986, 19, 1535.

(50) Kim, U.-J.; Park, J.; Li, C.; Jin, H.-J.; Valluzzi, R.; Kaplan, D. L., Structure and Properties of Silk Hydrogels. Biomacromolecules 2004, 5, 786-792, DOI: $10.1021 / \mathrm{bm} 0345460$.

(51) Wilson, D.; Valluzzi, R.; Kaplan, D., Conformational Transitions in Model Silk Peptides. Biophys. J. 2000, 78, 2690-2701, DOI: 10.1016/S0006-3495(00)76813-5.

(52) Hu, X.; Kaplan, D.; Cebe, P., Determining Beta-Sheet Crystallinity in Fibrous Proteins by Thermal Analysis and Infrared Spectroscopy. Macromolecules 2006, 39, 61616170, DOI: 10.1021/ma0610109.

(53) Wang, C.; Jiang, L.; Wei, D.; Li, Y.; Sui, X.; Wang, Z.; Li, D., Effect of secondary structure determined by FTIR spectra on surface hydrophobicity of soybean protein isolate. Procedia Eng. 2011, 15, 4819-4827, DOI: 10.1016/j.proeng.2011.08.900.

(54) Yan, C.; Pochan, D. J., Rheological properties of peptide-based hydrogels for biomedical and other applications. Chem. Soc. Rev. 2010, 39, 3528-3540, DOI: 10.1039/B919449P.

(55) Sim, H. G.; Ahn, K. H.; Lee, S. J., Large amplitude oscillatory shear behavior of complex fluids investigated by a network model: a guideline for classification. J. NonNewtonian Fluid Mech. 2003, 112, 237-250, DOI: 10.1016/S0377-0257(03)00102-2.

(56) Luo, K.; Yang, Y.; Shao, Z., Physically Crosslinked Biocompatible Silk-FibroinBased Hydrogels with High Mechanical Performance. Adv. Funct. Mater. 2016, 26, 872-880, DOI: $10.1002 / \mathrm{adfm} .201503450$.

(57) Han, J.; Lei, T.; Wu, Q., Facile preparation of mouldable polyvinyl alcohol-borax hydrogels reinforced by well-dispersed cellulose nanoparticles: physical, viscoelastic and mechanical properties. Cellulose, 2013, 20, 2947-2958, DOI: 10.1007/s10570-013-0082-5.

(58) Zhu, W.; Mow, V. C.; Koob, T. J.; Eyre, D. R., Viscoelastic shear properties of articular cartilage and the effects of glycosidase treatments. J. Orthop. Res. 1993, 11, 771781, DOI: $10.1002 /$ jor.1100110602.

(59) Galeski, A., Strength and toughness of crystalline polymer systems. Prog. Polym. Sci. 2003, 28, 1643-1699, DOI: 10.1016/j.progpolymsci.2003.09.003. 
(60) Thomas, V.; Zhang, X.; Catledge, S. A.; Vohra, Y. K., Functionally graded electrospun scaffolds with tunable mechanical properties for vascular tissue regeneration. Biomed. Mater. 2007, 2, 224-232, DOI: 10.1088/1748-6041/2/4/004.

(61) Yang, J.; Xu, F., Synergistic Reinforcing Mechanisms in Cellulose Nanofibrils Composite Hydrogels: Interfacial Dynamics, Energy Dissipation, and Damage Resistance. Biomacromolecules 2017, 18, 2623-2632, DOI: 10.1021/acs.biomac.7b00730.

(62) Wei, J.; Wang, J.; Su, S.; Wang, S.; Qiu, J.; Zhang, Z.; Christopher, G.; Ning, F.; Cong, W., 3D printing of an extremely tough hydrogel. RSC Adv. 2015, 5, 81324-81329, DOI: $10.1039 / \mathrm{C} 5 \mathrm{RA} 16362 \mathrm{E}$.

(63) Griffin, M.; Premakumar, Y.; Seifalian, A.; Butler, P. E.; Szarko, M., Biomechanical Characterization of Human Soft Tissues Using Indentation and Tensile Testing. J. Vis. Exp. 2016, 118, e54872, DOI: 10.3791/54872 (2016).

(64) Chia, H. N.; Hull, M. L., Compressive moduli of the human medial meniscus in the axial and radial directions at equilibrium and at a physiological strain rate. J. Orthop. Res. 2008, 26, 951-956, DOI: 10.1002/jor.20573.

(65) Umehara, S.; Tadano, S.; Abumi, K.; Katagiri, K.; Kaneda, K.; Ukai, T., Effects of degeneration on the elastic modulus distribution in the lumbar intervertebral disc. Spine 1996, 21, 811-819.

(66) Tang, Z.; Chen, F.; Chen, Q.; Zhu, L.; Yan, X.; Chen, H.; Ren, B.; Yang, J.; Qin, G.; Zheng, J., The energy dissipation and Mullins effect of tough polymer/graphene oxide hybrid nanocomposite hydrogels. Polym. Chem. 2017, 8, 4659-4672, DOI: 10.1039/C7PY01068K.

(67) Lewandowska, S. M.; Rumiński, S. Cellular Response to Biocomposites. In Biomedical Composites; Ambrosio, L., Ed.; Woodhead Publishing, 2017; pp 335-356, DOI: 10.1016/B978-0-08-100752-5.00015-9.

(68) Menas, A. L.; Yanamala, N.; Farcas, M. T.; Russo, M.; Friend, S.; Fournier, P. M.; Star, A.; Iavicoli, I.; Shurin, G. V.; Vogel, U. B.; Fadeel, B.; Beezhold, D.; Kisin, E. R.; Shvedova, A. A., Fibrillar vs crystalline nanocellulose pulmonary epithelial cell responses: Cytotoxicity or inflammation. Chemosphere 2017, 171, 671-680, DOI: 10.1016/j.chemosphere.2016.12.105.

(69) Kim, G.-D.; Yang, H.; Park, H. R.; Park, C.-S.; Park, Y. S.; Lee, S. E., Evaluation of immunoreactivity of in vitro and in vivo models against bacterial synthesized cellulose to be used as a prosthetic biomaterial. BioChip J. 2013, 7, 201-209, DOI: 10.1007/s13206-0137302-9. 
(70) Murphy, C. M.; Haugh, M. G.; O'Brien, F. J., The effect of mean pore size on cell attachment, proliferation and migration in collagen-glycosaminoglycan scaffolds for bone tissue engineering. Biomaterials 2010, 31, 461-466, DOI: 10.1016/j.biomaterials.2009.09.063.

(71) Baysal, K.; Aroguz, A. Z.; Adiguzel, Z.; Baysal, B. M., Chitosan/alginate crosslinked hydrogels: Preparation, characterization and application for cell growth purposes. Int. J. Biol. Macromol.. 2013, 59, 342-348, DOI: 10.1016/j.ijbiomac.2013.04.073.

(72) Lee, J. T. Y.; Chow, K. L., SEM sample preparation for cells on 3D scaffolds by freeze-drying and HMDS. Scanning 2012, 34, 12-25, DOI: 10.1002/sca.20271.

(73) Li, Y.; Zhang, Y.; Shi, F.; Tao, L.; Wei, Y.; Wang, X., Modulus-regulated 3D-cell proliferation in an injectable self-healing hydrogel. Colloids Surf., B 2017, 149, 168-173, DOI: 10.1016/j.colsurfb.2016.10.021.

(74) Cui, H.; Nowicki, M.; Fisher, J. P.; Zhang, L. G., 3D Bioprinting for Organ Regeneration. Adv. Healthcare Mater. 2017, 6, 1601118, DOI: 10.1002/adhm.201601118.

(75) Donderwinkel, I.; van Hest, J. C. M.; Cameron, N. R., Bio-inks for 3D bioprinting: recent advances and future prospects. Polym. Chem. 2017, 8, 4451-4471, DOI: 10.1039/C7PY00826K.

(76) Dorishetty, P.; Balu, R.; Sreekumar, A.; de Campo, L.; Mata, J. P.; Choudhury, N. R.; Dutta, N. K., Robust and Tunable Hybrid Hydrogels from Photo-Cross-Linked Soy Protein Isolate and Regenerated Silk Fibroin. ACS Sustainable Chem. Eng. 2019, 7, 9257-9271, DOI: 10.1021/acssuschemeng.9b00147.

(77) Bjork, J. W.; Johnson, S. L.; Tranquillo, R. T., Ruthenium-catalyzed photo crosslinking of fibrin-based engineered tissue. Biomaterials 2011, 32, 2479-2488, DOI: 10.1016/j.biomaterials.2010.12.010.

(78) Moberg, T.; Sahlin, K.; Yao, K.; Geng, S.; Westman, G.; Zhou, Q.; Oksman, K.; Rigdahl, M., Rheological properties of nanocellulose suspensions: effects of fibril/particle dimensions and surface characteristics. Cellulose 2017, 24, 2499-2510, DOI: 10.1007/s10570-017-1283-0.

(79) Kyle, S.; Jessop, Z. M.; Al-Sabah, A.; Whitaker, I. S., 'Printability' of Candidate Biomaterials for Extrusion Based 3D Printing: State-of-the-Art. Adv. Healthcare Mater. 2017, 6, 1700264, DOI: 10.1002/adhm.201700264.

(80) M'Barki, A.; Bocquet, L.; Stevenson, A., Linking Rheology and Printability for Dense and Strong Ceramics by Direct Ink Writing. Sci. Rep. 2017, 7, 6017, DOI: $10.1038 / \mathrm{s} 41598-017-06115-0$ 
(81) Zhang, J.; Xiao, P., 3D printing of photopolymers. Polym. Chem. 2018, 9, 1530-1540, DOI: $10.1039 / \mathrm{C} 8 \mathrm{PY} 00157 \mathrm{~J}$.

(82) Lee, B. H.; Lum, N.; Seow, L. Y.; Lim, P. Q.; Tan, L. P., Synthesis and Characterization of Types A and B Gelatin Methacryloyl for Bioink Applications. Materials 2016, 9, 797, DOI: 10.3390/ma9100797

(83) M.K. Hausmann, P.A. Rühs, G. Siqueira, J. Läuger, R. Libanori, T. Zimmermann, A.R. Studart., Dynamics of Cellulose Nanocrystal Alignment during 3D Printing. ACS Nano 2018, 12, 6926-6937, DOI: 10.1021/acsnano.8b02366 


\section{SUPPORTING INFORMATION}

\section{X-ray diffraction (XRD) analysis of the different types of nanocellulose used:}

Figure S1 (A) shows the XRD pattern of cellulose nanocrystals (CNC), cellulose nanofibers $(\mathrm{CNF})$ and bacterial nanocellulose $(\mathrm{BC})$. The cellulose I characteristics peaks were observed at $2 \theta=15.0^{\circ}, 16.5^{\circ}, 20.5^{\circ}$ and $22.5^{\circ}$, which corresponds to planes $(\overline{1} 10),(110),(102)$ and (200), respectively. Conversely, the cellulose II characteristics peaks were observed at $2 \theta=$ $12.5^{\circ}$ and $20.0^{\circ}$, which corresponds to planes $(\overline{1} 10)$ and (110), respectively. ${ }^{1}$ Cellulose I, which is the native form of cellulose has the highest axial elastic modulus, whereas cellulose II is generally formed by regeneration. ${ }^{2}$ The XRD data of CNC was deconvoluted with Gaussian curve fitting (Figure S1 (B)) using the MagicPlot software. The above $2 \theta$ values and an additional $2 \theta$ peak at $18.0^{\circ}$ (amorphous contribution) ${ }^{3}$ were used for the deconvolution.

\section{Small angle neutron scattering (SANS) analysis of nanocellulose dispersions:}

The SANS curves (Figure S2 (A)) of CNC, CNF and BC dispersions in $\mathrm{D}_{2} \mathrm{O}$ showed three distinctive regions: a high- $q$ Porod region, a mid- $q$ Guinier region, and a low- $q$ Porod-like region, which can also be clearly seen from Kratky plot (Figure S2 (B)). The high- $q$ Porod slope of 3.0, 2.8 and 2.5 obtained (using power law fit) for $\mathrm{CNC}, \mathrm{CNF}$ and $\mathrm{BC}$ can be attributed to the concentration fluctuations in nanocellulose fibrils or ribbons; where lower the slope value higher the fluctuations. ${ }^{4}$ The mean inter-particle distance (d) of CNC in dispersion was estimated as $\sim 67.0 \mathrm{~nm}$ from the correlation peak value using the relation $\mathrm{d}=$ $2 \pi /$ q $^{5}$ However, the SANS data of CNC dispersion could not be fit with a simple rigid rod model, considering the polydispersity of the system. Instead, the parallelepiped model with three characteristic dimensions: particle length, thickness and width were considered suitable based on literature reports. ${ }^{6}$ The parallelepiped model fit to SANS data of CNC is shown in Figure S2 (C). On the other hand, the SANS data of CNF and BC were fit (at mid- $q$ to high- 
q) with the flexible cylinder model - B(q) for intrinsic structure, and the power law function - A(q) for aggregation or network or entanglements at low- $q$, as shown in Figure S2 (D) and Figure S2 (E). ${ }^{7}$ The combined form factor model function used for CNF and BC SANS data fitting is given in equation $\mathrm{S} 1$ :

$$
I(q)=A(q)+B(q) \ldots(\mathrm{S} 1)
$$

where $\mathrm{I}(q)$ is the overall intensity of scattering. The length of the flexible cylinders was considered infinitively long for the fit based on atomic force microscopy results and the observed trend of scattering (increasing intensity) towards low- $q$. The obtained data fit model parameters were used for deconvolution of regenerated silk fibroin (RSF)/nanocellulose composite hydrogel SANS data.
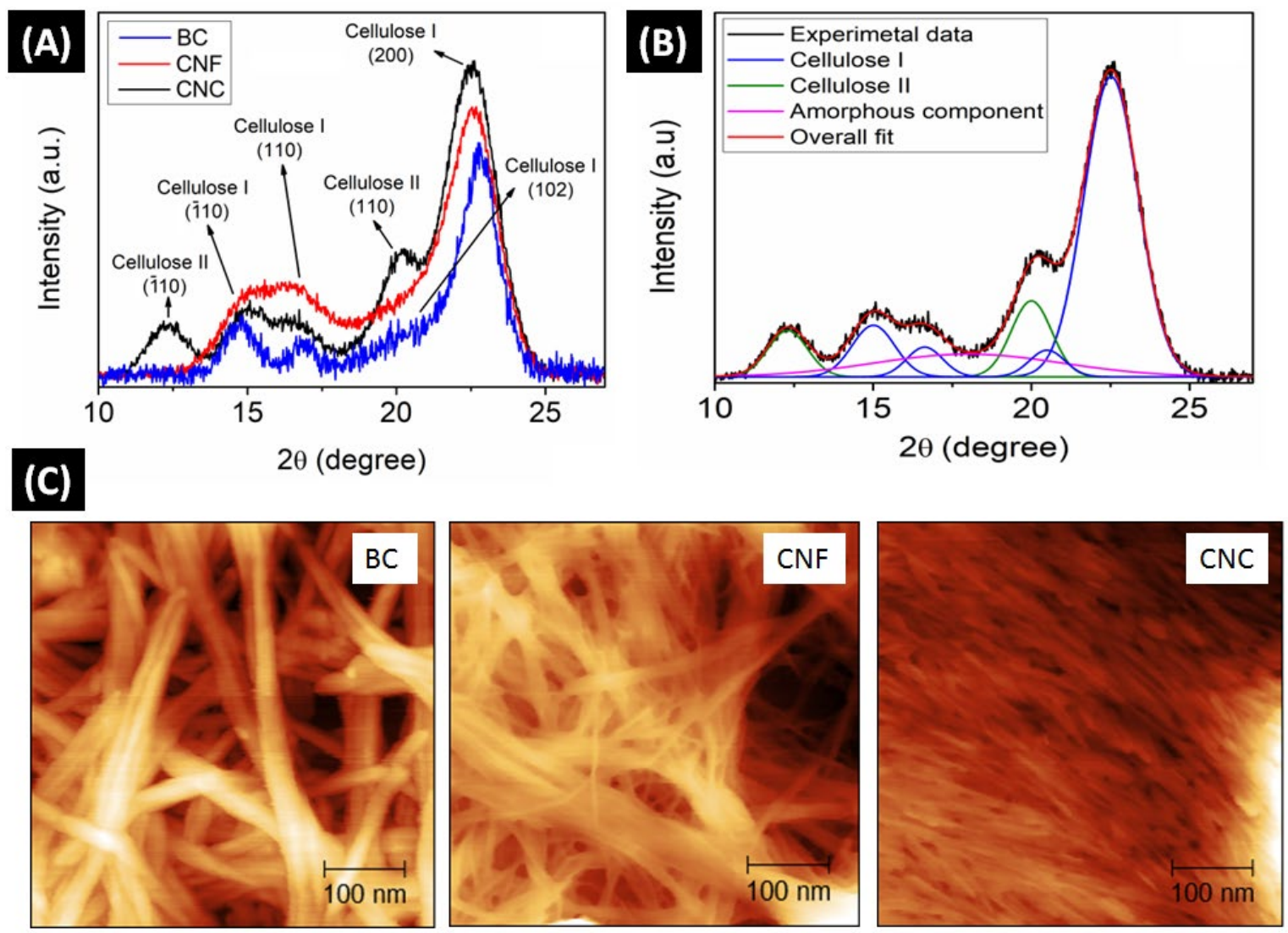

Figure S1. (A) XRD pattern of nanocelluloses. (B) Deconvoluted X-ray diffractogram of CNC. (C) AFM images of nanocelluloses. 

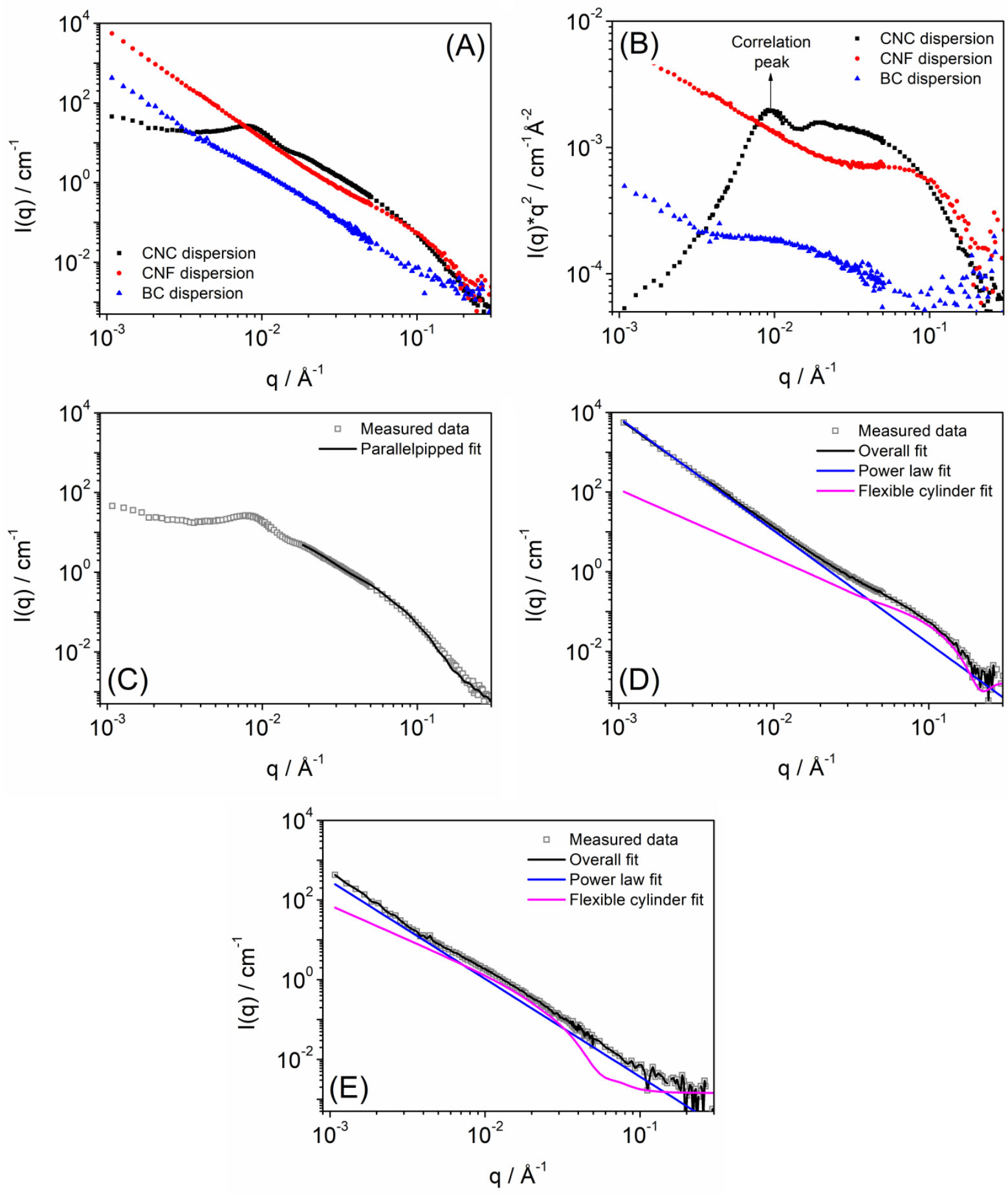

Figure S2. (A) SANS intensity profile and (B) Kratky plot of nanocellulose dispersion in $\mathrm{D}_{2} \mathrm{O}$. (C) to (E) are selective model function fits to the respective SANS data. 

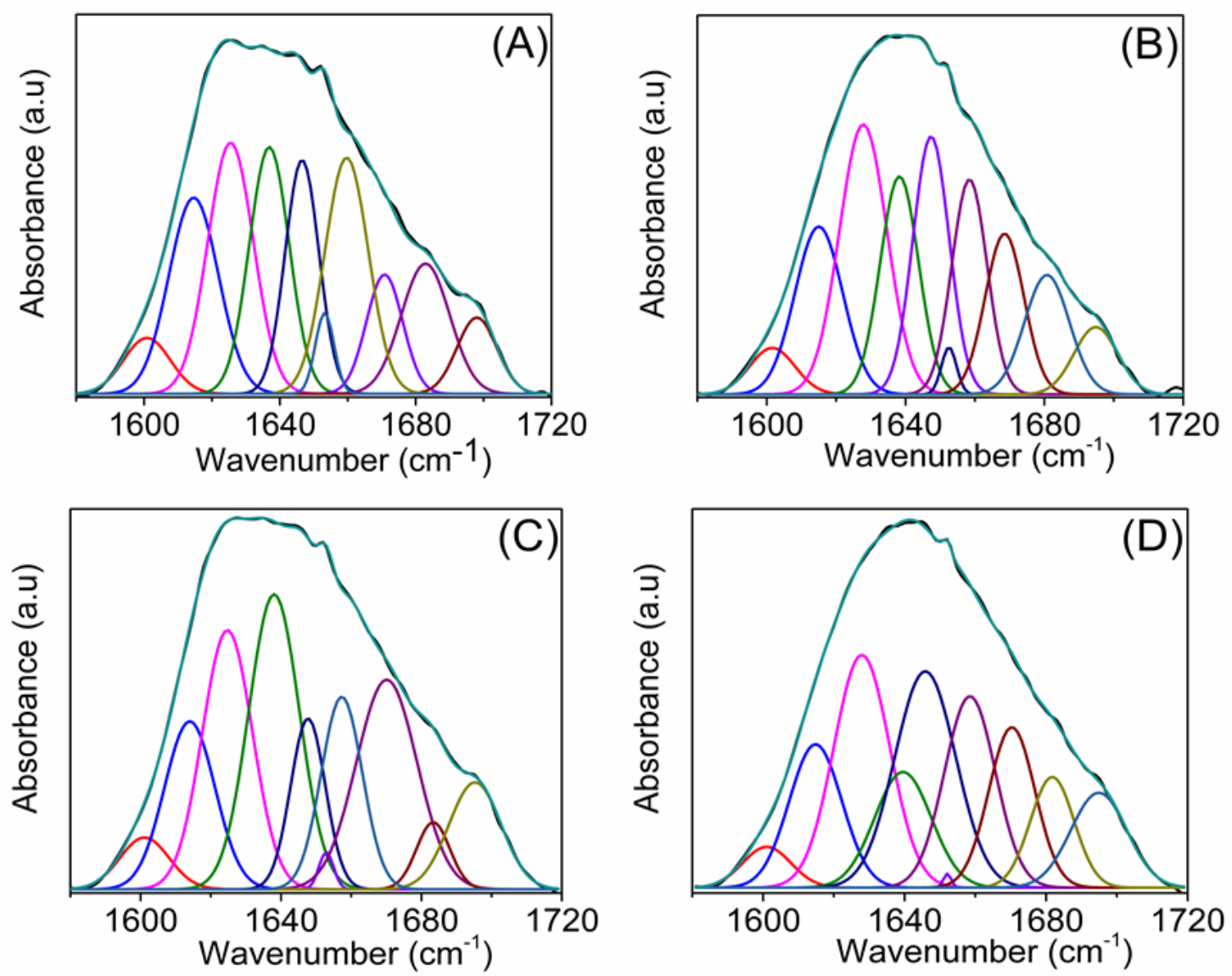

Figure S3. Deconvoluted FTIR spectra of (A) pristine regenerated silk fibroin (RSF), (B) RSF/CNC composite, (C) RSF/CNF composite and (D) RSF/BC composite hydrogels. 

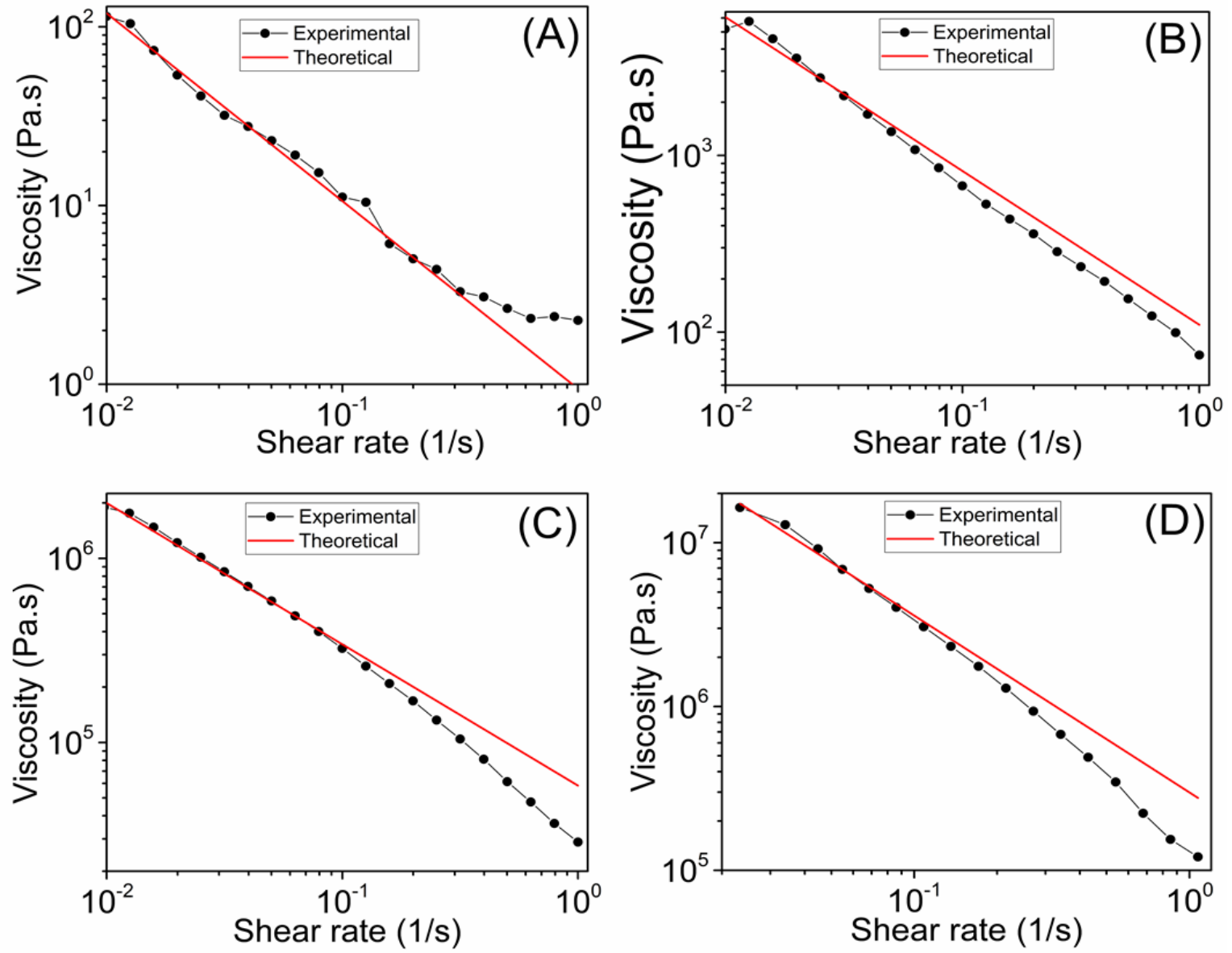

Figure S4. Experimental and theoretical (power law) fit of viscosity versus shear rate data of

(A) pristine RSF, (B) RSF/CNC composite, (C) RSF/CNF composite and (D) RSF/BC composite inks.

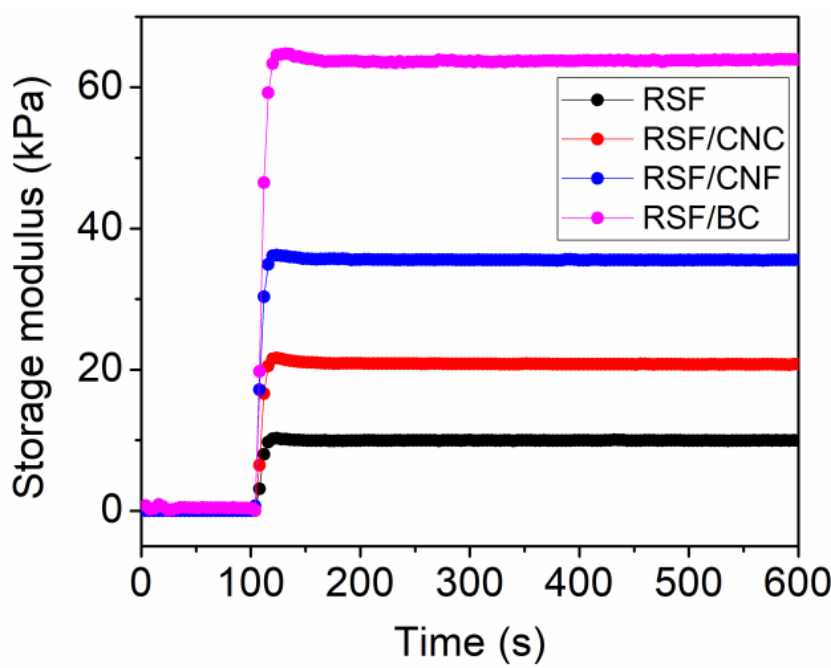

Figure S5. Dynamic oscillatory photocuring rheology showing crosslinking reaction kinetics of pristine RSF and RSF/nanocellulose composite inks. The lamp was turned on at $100 \mathrm{sec}$. 


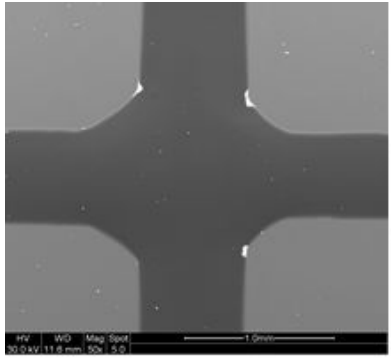

(a)

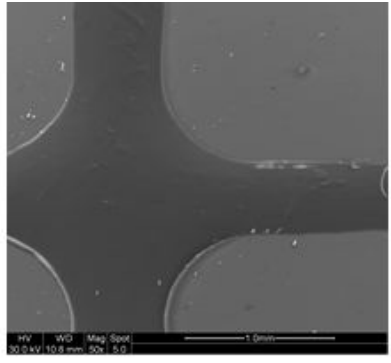

(b)

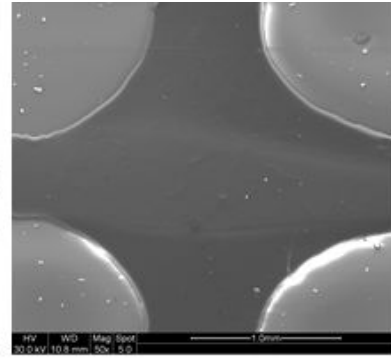

(c)

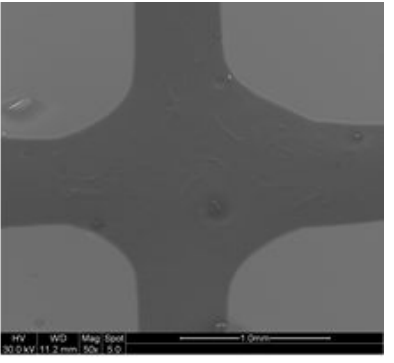

(d)

Figure S6. SEM micrographs (scale bar $-1 \mathrm{~mm}$ ) of 3D printed and equilibrium water swollen hydrogels; where (a) is pristine RSF, (b) is RSF/CNC composite, (c) is RSF/CNF composite and (d) is RSF/BC composite hydrogels.

Table S1. Estimated flow parameters of pristine RSF and RSF/nanocellulose composite inks.

\begin{tabular}{cccc}
\hline Sample & $\mathbf{K}$ & $\mathbf{n}$ & $\mathbf{R}^{\mathbf{2}}$ \\
\hline $\mathrm{RSF}$ & 0.94 & 0.05 & 0.99 \\
$\mathrm{RSF} / \mathrm{CNC}$ & 109.92 & 0.12 & 0.96 \\
$\mathrm{RSF} / \mathrm{CNF}$ & 4895.05 & 0.23 & 0.99 \\
$\mathrm{RSF} / \mathrm{BC}$ & 182073.72 & 0.34 & 0.98 \\
\hline
\end{tabular}

Table S2. Overall order of the properties of fabricated biomimetic hydrogel system.

\begin{tabular}{lc}
\hline Property & \multicolumn{1}{c}{ Sample order } \\
\hline Inter-hydrophobic domain distance & $\mathrm{RSF}>\mathrm{RSF} / \mathrm{BC}>\mathrm{RSF} / \mathrm{CNF}>\mathrm{RSF} / \mathrm{CNC}$ \\
\hline Water uptake & $\mathrm{RSF}>\mathrm{RSF} / \mathrm{BC}>\mathrm{RSF} / \mathrm{CNF}>\mathrm{RSF} / \mathrm{CNC}$ \\
\hline Shear storage modulus & $\mathrm{RSF} / \mathrm{BC}>\mathrm{RSF} / \mathrm{CNC}>\mathrm{RSF} / \mathrm{CNF}>\mathrm{RSF}$ \\
Young's Modulus & \\
\hline Tensile toughness & $\mathrm{RSF} / \mathrm{BC}>\mathrm{RSF} / \mathrm{CNF}>\mathrm{RSF} / \mathrm{CNC}>\mathrm{RSF}$ \\
Compression Modulus & \\
Energy dissipation & \\
\hline Biocompatibility & $\mathrm{RSF} / \mathrm{CNF}>\mathrm{RSF} / \mathrm{BC}>\mathrm{RSF}>\mathrm{RSF} / \mathrm{CNC}$ \\
\hline 3D printability & $\mathrm{RSF} / \mathrm{CNC}>\mathrm{RSF} / \mathrm{BC}>\mathrm{RSF}>\mathrm{RSF} / \mathrm{CFC}$
\end{tabular}




\section{References:}

(1) Novo, L. P.; Bras, J.; García, A.; Belgacem, N.; Curvelo, A. A. S., Subcritical Water: A Method for Green Production of Cellulose Nanocrystals. ACS Sustainable Chem. Eng. 2015, 3, 2839-2846, DOI: 10.1021/acssuschemeng.5b00762.

(2) Moon, R. J.; Martini, A.; Nairn, J.; Simonsen, J.; Youngblood, J., Cellulose nanomaterials review: structure, properties and nanocomposites. Chem. Soc. Rev. 2011, 40, 3941-3994, DOI: 10.1039/C0CS00108B.

(3) Park, S.; Baker, J. O.; Himmel, M. E.; Parilla, P. A.; Johnson, D. K., Cellulose crystallinity index: measurement techniques and their impact on interpreting cellulase performance. Biotechnol. Biofuels 2010, 3, 10, DOI: 10.1186/1754-6834-3-10.

(4) Koizumi, S.; Yue, Z.; Tomita, Y.; Kondo, T.; Iwase, H.; Yamaguchi, D.; Hashimoto, T., Bacterium organizes hierarchical amorphous structure in microbial cellulose. Eur. Phys. J. E 2008, 2, 137-142, DOI: 10.1140/epje/i2007-10259-3.

(5) Glatter, Whittaker, J. L.; Balu, R.; Knott, R.; de Campo, L.; Mata, J. P.; Rehm, C.; Hill, A. J.; Dutta, N. K.; Roy Choudhury, N., Structural evolution of photocrosslinked silk fibroin and silk fibroin-based hybrid hydrogels: A small angle and ultra-small angle scattering investigation. Int. J. Biol. Macromol. 2018, 114, 998-1007, DOI: 10.1016/j.ijbiomac.2018.03.044.

(6) Mao, Y.; Liu, K.; Zhan, C.; Geng, L.; Chu, B.; Hsiao, B. S., Characterization of nanocellulose using small-angle neutron, X-ray, and dynamic light scattering techniques. $J$. Phys. Chem. B 2017, 121, 1340-1351, DOI: 10.1021/acs.jpcb.6b11425.

(7) Pedersen, J. S.; Schurtenberger, P., Scattering functions of semiflexible polymers with and without excluded volume effects. Macromolecules 1996, 29, 7602-7612, DOI: 10.1021/ma9607630. 